\title{
Theoretical Study of the Borono-Mannich Reaction with Pinacol Allenylboronate
}

\author{
Sebastián O. Simonetti ${ }^{* a}$ and Silvina C. Pellegrinet ${ }^{*, a}$ \\ Instituto de Química Rosario (CONICET), Facultad de Ciencias Bioquímicas y Farmacéuticas, \\ Universidad Nacional de Rosario, Suipacha 531, Rosario (2000), Argentina

\section{Supporting Information}

\section{List of Contents:}

- Computational Methods with full list of authors in the Gaussian 09 reference S2.

- Shapes and energies of FMOs of the reactants (Figure S1) S3.

- Cartesian coordinates, absolute energies including zero-points energy corrections, free energies and free energies in solution (in Hartrees) of all stationary points reported in this paper and values of the imaginary frequencies in all transition states. S4-S32. 


\section{Computational methods}

Conformational searches for the reactants, the transition structures (TSs) and the products were run to locate the global minima at the B3LYP/6-31G* level of theory. Initially, a large number of geometries were generated using the conformational search module of Hyperchem with the MM+ method. ${ }^{1}$ Selected structures were then successively reoptimized at the B3LYP/6-31G* and M062X/6-311+G** levels of theory. ${ }^{2}$ Geometries for all structures were fully optimized and normal mode analysis was used to confirm the nature of the stationary points and to evaluate the thermochemical properties. Reported thermochemical properties include zero-point energies (ZPEs) without scaling and were calculated at $1 \mathrm{~atm}$ and $298.15 \mathrm{~K}$. The molecular orbitals of the reactants were calculated to analyze the frontier orbital interactions. Intrinsic reaction coordinate (IRCs) calculations were run to verify the connectivity between reactants, TSs and products. To examine the more important interactions in the TSs we performed natural bond orbital calculations and Wiberg bond indexes (WBIs) were analyzed. To interpret the presence of hydrogen bonds and the energy value, Second Order Perturbation analysis was carried out of the transition state at different theory levels. Free energies in solution were computed on the structures optimized in the gas phase at the B3LYP/6-31G* and M062X/6-311+G** levels of theory with the polarizable continuum

model $(\mathrm{PCM})^{3}$ using methanol as solvent and Solvation Model based on Density (SMD) ${ }^{4}$ to observe the differences between the models $\left(\varepsilon_{\text {methanol }}=32.63\right)$.

1 Hyperchem Professional Release 7.52, Hypercube, Inc., 2005.

2 Gaussian 09, Revision D.01: M. J. Frisch, G. W. Trucks, H. B. Schlegel, G. E. Scuseria, M. A. Robb, J. R. Cheeseman,G. Scalmani, V. Barone, B. Mennucci, G. A. Petersson, H. Nakatsuji, M. Caricato, X. Li, H. P. Hratchian, A. F.

Izmaylov, J. Bloino, G. Zheng, J. L. Sonnenberg, M. Hada, M. Ehara, K. Toyota, R. Fukuda, J. Hasegawa, M. Ishida, T. Nakajima, Y. Honda, O. Kitao, H. Nakai, T. Vreven, J. A. Montgomery, Jr., J. E. Peralta, F. Ogliaro, M. Bearpark, J. J. Heyd, E. Brothers, K. N. Kudin, V. N. Staroverov, T. Keith, R. Kobayashi, J. Normand, K. Raghavachari, A.

Rendell, J. C. Burant, S. S. Iyengar, J. Tomasi, M. Cossi, N. Rega, J. M. Millam, M. Klene, J. E. Knox, J. B. Cross, V. Bakken, C. Adamo, J. Jaramillo, R. Gomperts, R. E. Stratmann, O. Yazyev, A. J. Austin, R. Cammi, C. Pomelli, J. W. Ochterski, R. L. Martin, K. Morokuma, V. G. Zakrzewski, G. A. Voth, P. Salvador, J. J. Dannenberg, S. Dapprich, A. D. Daniels, O. Farkas, J. B. Foresman, J. V. Ortiz, J. Cioslowski, and D. J. Fox, Gaussian, Inc., Wallingford CT, 2013 3 (a) Miertš, S.; Scrocco, E.; Tomasi, J. Chem. Phys. 1981, 55, 117-129. (b) B. Mennucci and J. Tomasi, J. Chem. Phys. 1997, 106, 5151-5158. (c) J. Tomasi, B., Mennucci and R. Cammi, Chem. Rev. 2005, 105, 2999-3094.

4 A. V. Marenich, C. J. Cramer, D. G. Truhlar, J. of Phys. Chem. B 2009, 113, 6378-6396. 
LUUMO 12+60: -0.0870

$\Delta \mathrm{eV}: 0.11 \mathrm{eV}$

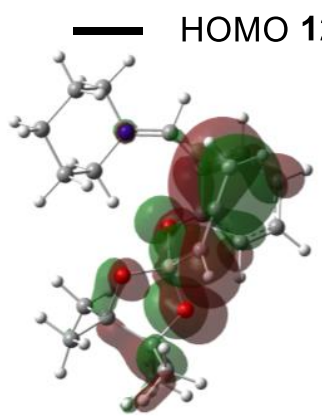

HOMO 12+60

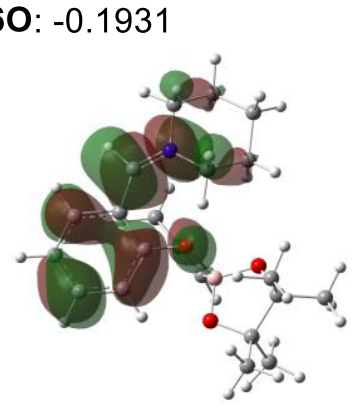

LUMO 12+60

- LUMO 10+60: -0.0873

$\Delta \mathrm{ev}: 0.11 \mathrm{ev}$

HOMO 10+60: -0.1983

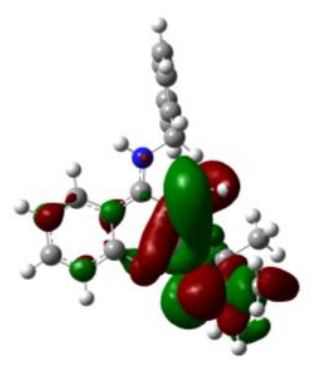

HOMO 10+6O<smiles>C=C[N+]1(Cc2ccccc2OB2OC(C)(C)C(C)(C)O2)CCCCC1</smiles>

$12+60$

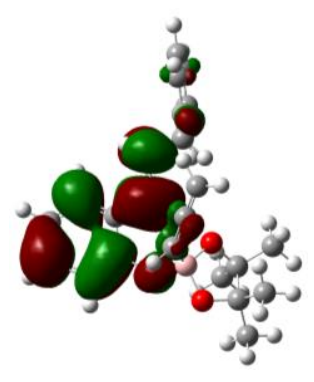

LUMO 10+60<smiles>C=C=C[B-]1(/[N+](=C/c2ccccc2O)Cc2ccccc2)OC(C)(C)C(C)(C)O1</smiles>

$10+6 N$

LUMO 10+6N: -0.06791

$\Delta \mathrm{eV}: 0.16 \mathrm{eV}$

HOMO 10+6N: -0.2267

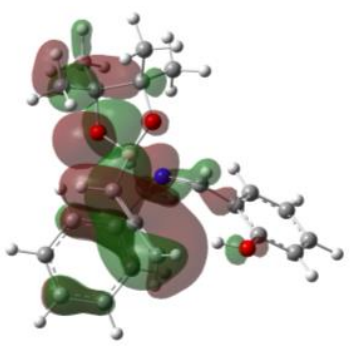

HOMO $10+6 N$

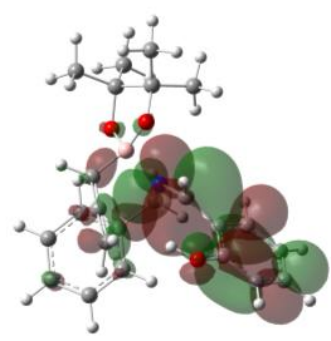

LUMO 10+6N

LUMO 14+6N: -0.0645

$\Delta \mathrm{ev}: 0.15 \mathrm{ev}$

HOMO 14+6N: -0.2107

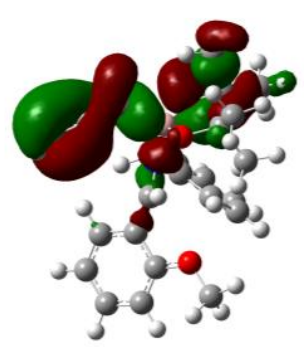

HOMO 14+6N<smiles>C=C(C=[NH+])O[B-]1(C=Cc2ccccc2Br)OC(C)(C)C(C)(C)O1</smiles>

$10+60$

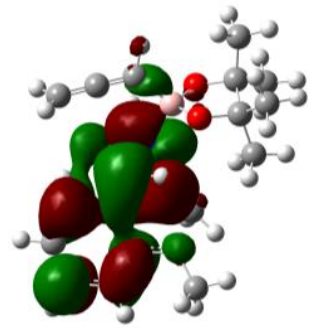

LUMO 14+6N<smiles>C=C(C)C=[N+](Cc1ccccc1OC)[B-]1(/C=C/c2ccccc2)OC(C)(C)C(C)(C)O1</smiles>

$14+6 N$

Figure S1. Shapes and energies of FMOs of the reactants $(12+60,10+6 \mathrm{~N}, 10+60$, and $14+6 \mathrm{~N})$. 


B3LYP/6-31G* Energy + ZPE: -1123.135475
B3LYP/6-31G* Free Energy: -1123.191102
M062X/6-311+G** Energy + ZPE: -1122.967069
M062X/6-311+G** Free Energy: -1123.022229
M062X/6-311+G** Free Energy in Methanol: -1123.070409
Number of Imaginary Frequencies: 0

$\begin{array}{lrrr}\mathrm{C} & -2.95309000 & 3.12952700 & -0.92474500 \\ \mathrm{C} & -2.77608800 & 1.76669200 & -0.85233700 \\ \mathrm{C} & -1.48542300 & 1.21989900 & -0.70852800 \\ \mathrm{C} & -0.34960100 & 2.06528800 & -0.59296000 \\ \mathrm{C} & -0.56047900 & 3.45926700 & -0.68744500 \\ \mathrm{C} & -1.82758400 & 3.97030900 & -0.84573700 \\ \mathrm{O} & 0.85934700 & 1.61592800 & -0.39869600 \\ \mathrm{C} & -1.19739200 & -0.18584600 & -0.75003200 \\ \mathrm{~N} & -1.85869300 & -1.15444000 & -0.19980700 \\ \mathrm{C} & -1.42618700 & -2.54863300 & -0.37127100 \\ \mathrm{C} & -2.60504400 & -3.38657900 & -0.86085300 \\ \mathrm{C} & -3.79952700 & -3.25473800 & 0.08844400 \\ \mathrm{C} & -4.16868800 & -1.78423300 & 0.30699500 \\ \mathrm{C} & -2.94880000 & -0.99179400 & 0.76866700 \\ \mathrm{~B} & 1.36820200 & 0.44009900 & 0.47225200 \\ \mathrm{O} & 1.47765400 & -0.77604500 & -0.36680500 \\ \mathrm{O} & 2.70941900 & 0.73536800 & 0.89202400 \\ \mathrm{C} & 0.36089400 & 0.20513900 & 1.74150500 \\ \mathrm{C} & -0.49271200 & 1.04532900 & 2.24957400 \\ \mathrm{C} & 2.81917800 & -1.24839200 & -0.29994500 \\ \mathrm{C} & 3.61123800 & 0.05500500 & 0.03131800 \\ \mathrm{C} & 3.86067300 & 0.90606400 & -1.21772100 \\ \mathrm{C} & 4.92166500 & -0.17473900 & 0.77064300 \\ \mathrm{C} & 2.91308900 & -2.27506900 & 0.83179300 \\ \mathrm{C} & 3.18829700 & -1.89978200 & -1.62533200 \\ \mathrm{C} & -1.35204100 & 1.92637700 & 2.69761700 \\ \mathrm{H} & -3.94110700 & 3.54825800 & -1.06573400 \\ \mathrm{H} & -3.62553800 & 1.10200300 & -0.97287800 \\ \mathrm{H} & 0.30846800 & 4.10003900 & -0.60598800 \\ \mathrm{H} & -1.96281200 & 5.04449200 & -0.90633700 \\ \mathrm{H} & -0.28850500 & -0.48953800 & -1.26598100 \\ \mathrm{H} & -1.07976600 & -2.89658100 & 0.60819600 \\ \mathrm{H} & -0.57525400 & -2.55942200 & -1.05115900 \\ \mathrm{H} & -2.28861400 & -4.42855600 & -0.94272400 \\ \mathrm{H} & -2.88610700 & -3.05003400 & -1.86424800 \\ \mathrm{H} & -3.54380300 & -3.70612600 & 1.05389900 \\ \mathrm{H} & -4.65651400 & -3.80529100 & -0.30456000 \\ \mathrm{H} & -4.95714800 & -1.68948600 & 1.05645200 \\ \mathrm{H} & -4.54778900 & -1.35103300 & -0.62519600 \\ \mathrm{H} & -3.14548100 & 0.06702300 & 0.90946800\end{array}$




$\begin{array}{lrrr}\mathrm{H} & -2.56734100 & -1.38730200 & 1.71717300 \\ \mathrm{H} & 0.47005800 & -0.75828000 & 2.24542700 \\ \mathrm{H} & 2.93688000 & 1.04375700 & -1.78218600 \\ \mathrm{H} & 4.20955300 & 1.88962400 & -0.89713800 \\ \mathrm{H} & 4.61853600 & 0.45830400 & -1.86672000 \\ \mathrm{H} & 5.59475400 & -0.80869800 & 0.18522900 \\ \mathrm{H} & 5.41428500 & 0.78520500 & 0.93913300 \\ \mathrm{H} & 4.74525000 & -0.64003800 & 1.74035200 \\ \mathrm{H} & 3.89933100 & -2.74416700 & 0.87644700 \\ \mathrm{H} & 2.70764100 & -1.80001900 & 1.79358400 \\ \mathrm{H} & 2.16633100 & -3.05536600 & 0.66044000 \\ \mathrm{H} & 2.60462400 & -2.81439800 & -1.76301800 \\ \mathrm{H} & 2.98084000 & -1.22873600 & -2.45910300 \\ \mathrm{H} & 4.24829900 & -2.17003700 & -1.64437100 \\ \mathrm{H} & -1.06069000 & 2.67221000 & 3.42859800 \\ \mathrm{H} & -2.37416900 & 1.95620000 & 2.33025400\end{array}$

$\mathrm{B} 3 \mathrm{LYP} / 6-31 \mathrm{G}^{*}$ Energy + ZPE: -1123.125094
$\mathrm{B} 3 \mathrm{LYP} / 6-31 \mathrm{G}^{*}$ Free Energy: -1123.177754
$\mathrm{M} 062 \mathrm{X} / 6-311+\mathrm{G}^{* *}$ Energy + ZPE: -1122.956274
$\mathrm{M} 062 \mathrm{X} / 6-311+\mathrm{G}^{* *}$ Free Energy: -1123.008131
$\mathrm{M} 062 \mathrm{X} / 6-31+\mathrm{G}^{* *}$ Free Energy in Methanol: -1123.048226
Number of Imaginary Frequencies: 1 (-422.92)

$\begin{array}{lrrr}\mathrm{C} & -2.44941000 & 3.30729300 & -1.13626400 \\ \mathrm{C} & -2.35236800 & 1.93747100 & -0.99144100 \\ \mathrm{C} & -1.17503900 & 1.32946200 & -0.52694000 \\ \mathrm{C} & -0.05566500 & 2.13177200 & -0.21645400 \\ \mathrm{C} & -0.17925100 & 3.52791300 & -0.34654600 \\ \mathrm{C} & -1.34818000 & 4.10262800 & -0.79911900 \\ \mathrm{O} & 1.09779300 & 1.66488300 & 0.22232700 \\ \mathrm{C} & -1.09051800 & -0.13715600 & -0.44873000 \\ \mathrm{~N} & -2.18533200 & -0.89349000 & -0.27406600 \\ \mathrm{C} & -2.09487600 & -2.32983300 & -0.53935100 \\ \mathrm{C} & -3.44742500 & -2.86252900 & -1.00351100 \\ \mathrm{C} & -4.53076500 & -2.55113100 & 0.03022300 \\ \mathrm{C} & -4.56185200 & -1.05310700 & 0.33831000 \\ \mathrm{C} & -3.17807100 & -0.55583300 & 0.74363200 \\ \mathrm{~B} & 1.43430100 & 0.24362900 & 0.38500800 \\ \mathrm{O} & 1.67572600 & -0.42399500 & -0.88414600 \\ \mathrm{O} & 2.59890100 & 0.04775100 & 1.18949800 \\ \mathrm{C} & 0.09559200 & -0.49273600 & 1.22069800 \\ \mathrm{C} & -0.43992400 & 0.15467400 & 2.22960000 \\ \mathrm{C} & 2.90711900 & -1.13710100 & -0.78444300\end{array}$




\begin{tabular}{lrrr}
$\mathrm{C}$ & 3.66244200 & -0.33396500 & 0.32056500 \\
$\mathrm{C}$ & 4.31659100 & 0.93132200 & -0.23985200 \\
$\mathrm{C}$ & 4.68144400 & -1.14369000 & 1.10782500 \\
$\mathrm{C}$ & 2.60561800 & -2.57221500 & -0.34416900 \\
$\mathrm{C}$ & 3.58982800 & -1.14471000 & -2.14380600 \\
$\mathrm{C}$ & -0.95097100 & 0.90832700 & 3.16131100 \\
$\mathrm{H}$ & -3.35890300 & 3.75390800 & -1.51709400 \\
$\mathrm{H}$ & -3.18517100 & 1.30240000 & -1.27543000 \\
$\mathrm{H}$ & 0.68476500 & 4.12633500 & -0.08525300 \\
$\mathrm{H}$ & -1.40786200 & 5.18022900 & -0.90148100 \\
$\mathrm{H}$ & -0.31809200 & -0.60352500 & -1.05716600 \\
$\mathrm{H}$ & -1.78924600 & -2.84381000 & 0.38390300 \\
$\mathrm{H}$ & -1.32158700 & -2.49142900 & -1.29108900 \\
$\mathrm{H}$ & -3.36902900 & -3.93906200 & -1.17148100 \\
$\mathrm{H}$ & -3.70025100 & -2.39569500 & -1.96086000 \\
$\mathrm{H}$ & -4.31738700 & -3.10381200 & 0.95268300 \\
$\mathrm{H}$ & -5.50640300 & -2.88810700 & -0.32617500 \\
$\mathrm{H}$ & -5.26767500 & -0.83806400 & 1.14369000 \\
$\mathrm{H}$ & -4.89340900 & -0.49831600 & -0.54581900 \\
$\mathrm{H}$ & -3.16701600 & 0.51968700 & 0.90951600 \\
$\mathrm{H}$ & -2.85524400 & -1.04128400 & 1.67988400 \\
$\mathrm{H}$ & 0.13630700 & -1.58043700 & 1.28027500 \\
$\mathrm{H}$ & 3.60223200 & 1.50266100 & -0.83622300 \\
$\mathrm{H}$ & 4.63512000 & 1.55426700 & 0.59808000 \\
$\mathrm{H}$ & 5.18925000 & 0.69426300 & -0.85424000 \\
$\mathrm{H}$ & 5.44899100 & -1.55346600 & 0.44436900 \\
$\mathrm{H}$ & 5.17048200 & -0.49715100 & 1.83930800 \\
$\mathrm{H}$ & 4.20214600 & -1.96202500 & 1.64555800 \\
$\mathrm{H}$ & 3.50333700 & -3.19547600 & -0.35221100 \\
$\mathrm{H}$ & 2.18420500 & -2.58666000 & 0.66335800 \\
$\mathrm{H}$ & 1.87910500 & -3.00437700 & -1.03730900 \\
$\mathrm{H}$ & 3.00889000 & -1.74996700 & -2.84383900 \\
$\mathrm{H}$ & 3.66551200 & -0.13502000 & -2.54618500 \\
$\mathrm{H}$ & 4.59294000 & -1.57505100 & -2.06955500 \\
$\mathrm{H}$ & -0.32427300 & 1.29417800 & 3.95812800 \\
$\mathrm{H}$ & -2.00119400 & 1.18083700 & 3.15539900 \\
& & & \\
\hline & & & \\
& & &
\end{tabular}

B3LYP/6-31G* Energy + ZPE: -1123.112164
B3LYP/6-31G* Free Energy: -1123.164389
M062X/6-311+G** Energy + ZPE: -1122.944821
M062X/6-311+G** Free Energy: -1122.996169
M062X/6-31+G** Free Energy in Methanol: -1123.034153
Number of Imaginary Frequencies: 1 (-512.95)

$\begin{array}{llll}\text { C } & -1.78853900 & 4.08307900 & -0.51739700\end{array}$ 


\begin{tabular}{|c|c|c|c|}
\hline $\mathrm{C}$ & -2.52395900 & 2.95838800 & -0.16883300 \\
\hline $\mathrm{C}$ & -1.92007900 & 1.70261800 & -0.08798000 \\
\hline $\mathrm{C}$ & -0.54596900 & 1.57151000 & -0.35796200 \\
\hline $\mathrm{C}$ & 0.19376000 & 2.70938800 & -0.69469100 \\
\hline $\mathrm{C}$ & -0.42590700 & 3.94927700 & -0.77044400 \\
\hline $\mathrm{O}$ & -0.01015400 & 0.34277800 & -0.33548600 \\
\hline $\mathrm{C}$ & -2.75517300 & 0.55938200 & 0.38213000 \\
\hline $\mathrm{N}$ & -2.98678000 & -0.52919800 & -0.38543600 \\
\hline $\mathrm{C}$ & -3.93384800 & -1.53391600 & 0.08778900 \\
\hline $\mathrm{C}$ & -3.20229400 & -2.81959000 & 0.48894800 \\
\hline $\mathrm{C}$ & -2.37326200 & -3.34551300 & -0.68524900 \\
\hline $\mathrm{C}$ & -1.41707200 & -2.26285800 & -1.18977200 \\
\hline $\mathrm{C}$ & -2.18996400 & -0.98918500 & -1.52502800 \\
\hline B & 1.13513000 & -0.05269400 & 0.50438800 \\
\hline $\mathrm{O}$ & 2.22366300 & 0.88276800 & 0.51327100 \\
\hline $\mathrm{O}$ & 1.64956700 & -1.33286900 & 0.11649800 \\
\hline $\mathrm{C}$ & 0.49920300 & -0.22487400 & 2.13053200 \\
\hline $\mathrm{C}$ & -0.74770500 & -0.08941700 & 2.25160000 \\
\hline $\mathrm{C}$ & 3.43062500 & 0.15196300 & 0.29876000 \\
\hline $\mathrm{C}$ & 2.92423800 & -1.10222100 & -0.48391300 \\
\hline $\mathrm{C}$ & 3.78682200 & -2.34343900 & -0.31784600 \\
\hline $\mathrm{C}$ & 2.71349200 & -0.80336700 & -1.97070200 \\
\hline $\mathrm{C}$ & 4.03235900 & -0.22245500 & 1.65490500 \\
\hline $\mathrm{C}$ & 4.40547900 & 1.02920100 & -0.47290400 \\
\hline $\mathrm{C}$ & -2.09752300 & 0.14865700 & 2.19941200 \\
\hline $\mathrm{H}$ & -2.26938400 & 5.05105800 & -0.58104600 \\
\hline $\mathrm{H}$ & -3.58332700 & 3.05216200 & 0.04909600 \\
\hline $\mathrm{H}$ & 1.25451300 & 2.59070900 & -0.87130100 \\
\hline $\mathrm{H}$ & 0.16260900 & 4.82174500 & -1.03083700 \\
\hline $\mathrm{H}$ & -3.67297000 & 0.90246900 & 0.85656300 \\
\hline $\mathrm{H}$ & -4.63549300 & -1.75382600 & -0.72635300 \\
\hline $\mathrm{H}$ & -4.50745400 & -1.11789600 & 0.91876300 \\
\hline $\mathrm{H}$ & -3.93502800 & -3.56108100 & 0.81897500 \\
\hline $\mathrm{H}$ & -2.53682900 & -2.61213700 & 1.33358800 \\
\hline $\mathrm{H}$ & -3.05007100 & -3.64831300 & -1.49431700 \\
\hline $\mathrm{H}$ & -1.81651800 & -4.23539200 & -0.38375600 \\
\hline $\mathrm{H}$ & -0.88959200 & -2.60048000 & -2.08595200 \\
\hline $\mathrm{H}$ & -0.65541700 & -2.03324500 & -0.44078700 \\
\hline $\mathrm{H}$ & -1.53339000 & -0.19288600 & -1.85688900 \\
\hline $\mathrm{H}$ & -2.91093900 & -1.19977400 & -2.32663400 \\
\hline $\mathrm{H}$ & 1.22412800 & -0.42865400 & 2.90661400 \\
\hline $\mathrm{H}$ & 3.81593700 & -2.66356100 & 0.72362600 \\
\hline $\mathrm{H}$ & 3.37098900 & -3.15887000 & -0.91365600 \\
\hline $\mathrm{H}$ & 4.80845000 & -2.15433500 & -0.66072500 \\
\hline $\mathrm{H}$ & 3.66398700 & -0.68773200 & -2.49783500 \\
\hline $\mathrm{H}$ & 2.16611600 & -1.63611600 & -2.41740400 \\
\hline $\mathrm{H}$ & 2.11993100 & 0.10442500 & -2.10070500 \\
\hline $\mathrm{H}$ & 3.38930200 & -0.92646500 & 2.18716300 \\
\hline $\mathrm{H}$ & 5.01928200 & -0.67825000 & 1.54190900 \\
\hline $\mathrm{H}$ & 4.13150100 & 0.68473800 & 2.25468000 \\
\hline $\mathrm{H}$ & 4.71797500 & 1.86738600 & 0.15397800 \\
\hline
\end{tabular}




$\begin{array}{lrrr}\mathrm{H} & 5.29626800 & 0.46124300 & -0.75741300 \\ \mathrm{H} & 3.94128300 & 1.43041400 & -1.37409300 \\ \mathrm{H} & -2.74176800 & -0.69609100 & 2.42913500 \\ \mathrm{H} & -2.40499800 & 1.08541300 & 2.65933400\end{array}$

B3LYP/6-31G* Energy + ZPE: -1123.180359
B3LYP/6-31G* Free Energy: -1123.237461
M062X/6-311+G** Energy + ZPE: -1123.014673
M062X/6-311+G** Free Energy: -1123.069315
M062X/6-31+G** Free Energy in Methanol: -1123.099543
Number of Imaginary Frequencies: 0

$\begin{array}{lrrr}\mathrm{C} & -2.07935900 & 3.17271900 & -1.51842100 \\ \mathrm{C} & -2.09886300 & 1.85679200 & -1.07446600 \\ \mathrm{C} & -1.03866200 & 1.29594100 & -0.35734100 \\ \mathrm{C} & 0.07984100 & 2.11283700 & -0.13810600 \\ \mathrm{C} & 0.10369700 & 3.43519000 & -0.56981300 \\ \mathrm{C} & -0.97351600 & 3.97209000 & -1.25727800 \\ \mathrm{O} & 1.19399300 & 1.69784600 & 0.55619000 \\ \mathrm{C} & -1.09731700 & -0.16651900 & 0.08100700 \\ \mathrm{~N} & -2.39951900 & -0.77258900 & -0.20788900 \\ \mathrm{C} & -2.33501600 & -2.21467300 & -0.41181300 \\ \mathrm{C} & -3.65588600 & -2.72947600 & -0.97920200 \\ \mathrm{C} & -4.82282700 & -2.35077200 & -0.06718000 \\ \mathrm{C} & -4.81159000 & -0.84602100 & 0.20393100 \\ \mathrm{C} & -3.45104900 & -0.42062000 & 0.74600600 \\ \mathrm{~B} & 1.97592000 & 0.64465700 & 0.21534800 \\ \mathrm{O} & 1.88773200 & -0.06083600 & -0.96038700 \\ \mathrm{O} & 2.98719900 & 0.21575200 & 1.03309300 \\ \mathrm{C} & -0.67991600 & -0.41084900 & 1.53055600 \\ \mathrm{C} & -0.59176100 & 0.50169900 & 2.45584500 \\ \mathrm{C} & 2.78203200 & -1.18975200 & -0.83173700 \\ \mathrm{C} & 3.79702800 & -0.68421800 & 0.24935400 \\ \mathrm{C} & 4.93146100 & 0.14043000 & -0.35342200 \\ \mathrm{C} & 4.35111200 & -1.76967100 & 1.15536200 \\ \mathrm{C} & 1.94814300 & -2.37238600 & -0.34609000 \\ \mathrm{C} & 3.39472300 & -1.49379500 & -2.18703300 \\ \mathrm{C} & -0.45909600 & 1.44716200 & 3.34099500 \\ \mathrm{H} & -2.92589300 & 3.56814700 & -2.06661200 \\ \mathrm{H} & -2.94938900 & 1.22503900 & -1.29640500 \\ \mathrm{H} & 0.98825300 & 4.02141200 & -0.35235700 \\ \mathrm{H} & -0.94330700 & 5.00234700 & -1.59131600 \\ \mathrm{H} & -0.37833500 & -0.69901900 & -0.54938200 \\ \mathrm{H} & -2.11471500 & -2.74930900 & 0.53193300\end{array}$




$\begin{array}{lrrr}\mathrm{H} & -1.52130400 & -2.42815000 & -1.11002300 \\ \mathrm{H} & -3.60080400 & -3.81392300 & -1.10825400 \\ \mathrm{H} & -3.80306900 & -2.28298300 & -1.96783900 \\ \mathrm{H} & -4.72441100 & -2.88538600 & 0.88563100 \\ \mathrm{H} & -5.77286400 & -2.65968200 & -0.51044000 \\ \mathrm{H} & -5.59175400 & -0.57696200 & 0.92096400 \\ \mathrm{H} & -5.01268100 & -0.30159700 & -0.72510600 \\ \mathrm{H} & -3.41873500 & 0.65732000 & 0.92211500 \\ \mathrm{H} & -3.27980500 & -0.90912000 & 1.72041400 \\ \mathrm{H} & -0.51305900 & -1.45001200 & 1.80685700 \\ \mathrm{H} & 4.54094900 & 0.91492900 & -1.01779600 \\ \mathrm{H} & 5.47714200 & 0.62771400 & 0.45606800 \\ \mathrm{H} & 5.62456300 & -0.48788000 & -0.91690400 \\ \mathrm{H} & 4.88401900 & -2.52186900 & 0.56723300 \\ \mathrm{H} & 5.05345300 & -1.32780200 & 1.86426500 \\ \mathrm{H} & 3.55776000 & -2.25784300 & 1.72077400 \\ \mathrm{H} & 2.55057200 & -3.28040400 & -0.27479000 \\ \mathrm{H} & 1.50860900 & -2.16387200 & 0.63252200 \\ \mathrm{H} & 1.14132000 & -2.54970000 & -1.06025100 \\ \mathrm{H} & 2.61926400 & -1.85057800 & -2.86724100 \\ \mathrm{H} & 3.85033800 & -0.60621100 & -2.62399400 \\ \mathrm{H} & 4.15514300 & -2.27397300 & -2.09271000 \\ \mathrm{H} & 0.50205500 & 1.93205200 & 3.47613900 \\ \mathrm{H} & -1.29675200 & 1.77341500 & 3.94689100\end{array}$

B3LYP/6-31G* Energy + ZPE: -1123.175130
B3LYP/6-31G* Free Energy: -1123.232029
M062X/6-311+G** Energy + ZPE: -1123.012872
M062X/6-311+G** Free Energy: -1123.068735
M062X/6-31+G** Free Energy in Methanol: -1123.102124
Number of Imaginary Frequencies: 0

$\begin{array}{lrrr}\mathrm{C} & -1.65428900 & 2.99116900 & -1.67497400 \\ \mathrm{C} & -2.02633700 & 2.22983300 & -0.57437600 \\ \mathrm{C} & -1.34539800 & 1.06178500 & -0.22683700 \\ \mathrm{C} & -0.25786600 & 0.69088400 & -1.02114300 \\ \mathrm{C} & 0.12921600 & 1.44120000 & -2.12620200 \\ \mathrm{C} & -0.57267600 & 2.59295000 & -2.45340200 \\ \mathrm{O} & 0.41126900 & -0.47169500 & -0.70337100 \\ \mathrm{C} & -1.77168500 & 0.19828200 & 0.95570800 \\ \mathrm{~N} & -2.90042800 & -0.69793700 & 0.68130600 \\ \mathrm{C} & -4.13448000 & -0.03914200 & 0.25907700 \\ \mathrm{C} & -5.28658300 & -1.04101900 & 0.22730000 \\ \mathrm{C} & -4.96175900 & -2.19831300 & -0.71504900 \\ \mathrm{C} & -3.62244900 & -2.82155600 & -0.32492900\end{array}$




$\begin{array}{lrrr}\mathrm{C} & -2.53286400 & -1.75686500 & -0.26056800 \\ \mathrm{C} & -2.03761000 & 1.03176200 & 2.22280600 \\ \mathrm{C} & -0.83169000 & 1.73615900 & 2.65508100 \\ \mathrm{C} & 0.17664800 & 2.29919700 & 2.98257900 \\ \mathrm{~B} & 1.74366800 & -0.49879800 & -0.42807500 \\ \mathrm{O} & 2.61239800 & 0.54362400 & -0.61826700 \\ \mathrm{C} & 3.83467600 & 0.17976900 & 0.06539000 \\ \mathrm{C} & 3.75714100 & -1.38518200 & 0.06569200 \\ \mathrm{O} & 2.33260600 & -1.61995100 & 0.08936500 \\ \mathrm{C} & 5.01669700 & 0.75831400 & -0.69053500 \\ \mathrm{C} & 3.74926100 & 0.77444800 & 1.46845000 \\ \mathrm{C} & 4.29290800 & -2.00258100 & -1.22283300 \\ \mathrm{C} & 4.38774100 & -2.04865100 & 1.27699200 \\ \mathrm{H} & -2.20236800 & 3.89241800 & -1.92094900 \\ \mathrm{H} & -2.86446100 & 2.55626200 & 0.03151800 \\ \mathrm{H} & 0.98457200 & 1.12040200 & -2.70807300 \\ \mathrm{H} & -0.27126800 & 3.17964600 & -3.31304700 \\ \mathrm{H} & -0.93519700 & -0.45837300 & 1.19860500 \\ \mathrm{H} & -4.02305100 & 0.41285500 & -0.74239300 \\ \mathrm{H} & -4.37050100 & 0.76636200 & 0.95897300 \\ \mathrm{H} & -6.20167000 & -0.53046400 & -0.08515000 \\ \mathrm{H} & -5.44682000 & -1.42434300 & 1.24014800 \\ \mathrm{H} & -4.89819200 & -1.81866600 & -1.74203200 \\ \mathrm{H} & -5.75854500 & -2.94638000 & -0.69767900 \\ \mathrm{H} & -3.33064300 & -3.59612000 & -1.03916000 \\ \mathrm{H} & -3.71012500 & -3.29238600 & 0.65961600 \\ \mathrm{H} & -1.58786000 & -2.19461700 & 0.06737500 \\ \mathrm{H} & -2.36406000 & -1.34215900 & -1.27152700 \\ \mathrm{H} & -2.83391900 & 1.76401300 & 2.07049000 \\ \mathrm{H} & -2.37330700 & 0.34752800 & 3.00605000 \\ \mathrm{H} & 1.06300500 & 2.80848200 & 3.27561900 \\ \mathrm{H} & 4.99182200 & 1.84757100 & -0.62687000 \\ \mathrm{H} & 5.95539300 & 0.40924300 & -0.25151900 \\ \mathrm{H} & 4.99231900 & 0.47540200 & -1.74239200 \\ \mathrm{H} & 2.90419500 & 0.35511500 & 2.01985000 \\ \mathrm{H} & 4.66594500 & 0.59277600 & 2.03354000 \\ \mathrm{H} & 3.59796700 & 1.85159700 & 1.37956800 \\ \mathrm{H} & 5.37940100 & -1.91224300 & -1.28533800 \\ \mathrm{H} & 4.02786600 & -3.06097200 & -1.23916200 \\ \mathrm{H} & 3.85003400 & -1.52493200 & -2.09990100 \\ \mathrm{H} & 3.89843000 & -1.73655500 & 2.19885700 \\ \mathrm{H} & 4.29300600 & -3.13239100 & 1.18848000 \\ & 5.45087800 & -1.79998900 & 1.33656000\end{array}$




B3LYP/6-31G* Energy + ZPE: -1198.153948
B3LYP/6-31G* Free Energy: -1198.211299
M062X/6-311+G** Energy + ZPE: -1197.990439
M062X/6-311+G** Free Energy: -1198.046230
M062X/6-31+G** Free Energy in Methanol: -1198.080458
Number of Imaginary Frequencies: 0

$\begin{array}{lrrr}\mathrm{C} & -2.91342500 & -3.47021200 & -1.05706400 \\ \mathrm{C} & -1.75054600 & -2.80276500 & -0.70488400 \\ \mathrm{C} & -1.77971700 & -1.76499800 & 0.23085900 \\ \mathrm{C} & -2.99531900 & -1.38876000 & 0.81184600 \\ \mathrm{C} & -4.16378900 & -2.06522300 & 0.45871800 \\ \mathrm{C} & -4.11959200 & -3.09678100 & -0.46520200 \\ \mathrm{C} & -0.51853000 & -1.11150200 & 0.65255900 \\ \mathrm{~N} & 0.17027800 & -0.30143200 & -0.04560400 \\ \mathrm{C} & -0.27739900 & 0.18422200 & -1.36668900 \\ \mathrm{C} & -1.09362600 & 1.44548200 & -1.19463300 \\ \mathrm{C} & -2.48572500 & 1.38124300 & -1.16089400 \\ \mathrm{C} & -3.23644100 & 2.53428900 & -0.94999700 \\ \mathrm{C} & -2.59577800 & 3.75585400 & -0.77343000 \\ \mathrm{C} & -1.20464100 & 3.82383600 & -0.81228100 \\ \mathrm{C} & -0.45419600 & 2.67306900 & -1.02247700 \\ \mathrm{O} & -3.10193300 & -0.38428700 & 1.70829200 \\ \mathrm{~B} & 1.58615500 & 0.29268700 & 0.61620700 \\ \mathrm{O} & 2.31493900 & -0.81847900 & 1.16885500 \\ \mathrm{O} & 2.35734100 & 0.81064300 & -0.49036000 \\ \mathrm{C} & 3.62584200 & 0.15190000 & -0.48710000 \\ \mathrm{C} & 3.29096900 & -1.20845600 & 0.20292000 \\ \mathrm{C} & 4.46226700 & -1.85932900 & 0.92229800 \\ \mathrm{C} & 2.66404600 & -2.20875300 & -0.77488200 \\ \mathrm{C} & 4.59873900 & 0.98922100 & 0.34448300 \\ \mathrm{C} & 4.12991900 & 0.03191900 & -1.91733300 \\ \mathrm{C} & 1.14697400 & 1.39134000 & 1.72227100 \\ \mathrm{C} & -0.05303000 & 1.68675400 & 2.11860100 \\ \mathrm{C} & -1.28547300 & 1.98613000 & 2.45818900 \\ \mathrm{H} & -2.88015400 & -4.27685100 & -1.77789700 \\ \mathrm{H} & -0.79854100 & -3.08770900 & -1.14147300 \\ \mathrm{H} & -5.09298200 & -1.75234400 & 0.91873800 \\ \mathrm{H} & -5.03466000 & -3.61245500 & -0.73103300 \\ \mathrm{H} & -0.11069200 & -1.34151600 & 1.63898200 \\ \mathrm{H} & 0.63105500 & 0.38651800 & -1.93224500 \\ \mathrm{H} & -0.86505300 & -0.58657700 & -1.86324400 \\ \mathrm{H} & -2.98518300 & 0.42796600 & -1.30721500 \\ \mathrm{H} & -4.31811600 & 2.47661000 & -0.92557400 \\ \mathrm{H} & -3.17742400 & 4.65522300 & -0.60785300 \\ \mathrm{H} & -0.70429800 & 4.77509700 & -0.67409400 \\ \mathrm{H} & 0.63062100 & 2.71014100 & -1.03470100\end{array}$




$\begin{array}{lrrr}\mathrm{H} & -2.34536200 & 0.22509500 & 1.68036500 \\ \mathrm{H} & 4.14689600 & -2.81557200 & 1.34543800 \\ \mathrm{H} & 5.28844600 & -2.04498500 & 0.22974300 \\ \mathrm{H} & 4.81480000 & -1.22685300 & 1.73631100 \\ \mathrm{H} & 1.88039800 & -1.73949000 & -1.37515500 \\ \mathrm{H} & 3.41025600 & -2.62657000 & -1.45497500 \\ \mathrm{H} & 2.22064700 & -3.02573600 & -0.20015500 \\ \mathrm{H} & 4.27551000 & 1.02869700 & 1.38706100 \\ \mathrm{H} & 5.61369800 & 0.58589400 & 0.30703700 \\ \mathrm{H} & 4.61114800 & 2.00549300 & -0.05395100 \\ \mathrm{H} & 4.34878100 & 1.02656200 & -2.31162000 \\ \mathrm{H} & 5.04853400 & -0.56088500 & -1.95578200 \\ \mathrm{H} & 3.38377500 & -0.43323200 & -2.56251400 \\ \mathrm{H} & 1.96251100 & 1.97192200 & 2.15589200 \\ \mathrm{H} & -1.75199400 & 1.54777000 & 3.33529300 \\ \mathrm{H} & -1.83725200 & 2.74045100 & 1.90326500\end{array}$

B3LYP/6-31G* Energy + ZPE: -1198.107451
B3LYP/6-31G* Free Energy: -1198.164339
M062X/6-311+G** Energy + ZPE: -1197.934260
M062X/6-311+G** Free Energy: -1197.991370
M062X/6-31+G** Free Energy in Methanol: -1198.024466
Number of Imaginary Frequencies: 1 (-530.94)

$\begin{array}{lrrr}\mathrm{C} & 2.63467600 & 3.61915500 & 1.04150900 \\ \mathrm{C} & 2.01784100 & 2.42479100 & 1.33990900 \\ \mathrm{C} & 1.19593000 & 1.75529700 & 0.41372900 \\ \mathrm{C} & 0.96150200 & 2.35215300 & -0.84703600 \\ \mathrm{C} & 1.62768600 & 3.55050500 & -1.15186800 \\ \mathrm{C} & 2.44349000 & 4.17318800 & -0.23009400 \\ \mathrm{C} & 0.76098600 & 0.40543800 & 0.79611100 \\ \mathrm{~N} & 0.19433500 & -0.49318700 & -0.07670900 \\ \mathrm{C} & 0.61696600 & -1.87666200 & 0.12794100 \\ \mathrm{C} & 2.07687500 & -2.08168900 & -0.21737500 \\ \mathrm{C} & 2.70665000 & -1.30414500 & -1.19038700 \\ \mathrm{C} & 4.04282900 & -1.52490100 & -1.50989700 \\ \mathrm{C} & 4.76605900 & -2.52112300 & -0.86115900 \\ \mathrm{C} & 4.14549200 & -3.29682600 & 0.11249900 \\ \mathrm{C} & 2.81102300 & -3.07321800 & 0.43371200 \\ \mathrm{O} & 0.18350800 & 1.85213900 & -1.80901400 \\ \mathrm{H} & 3.26788600 & 4.10930000 & 1.76931700 \\ \mathrm{H} & 2.17380100 & 1.96183600 & 2.30989600 \\ \mathrm{H} & 1.45635800 & 3.97508500 & -2.13338500 \\ \mathrm{H} & 2.93271600 & 5.10365300 & -0.49411700 \\ \mathrm{H} & 1.37948300 & -0.01635700 & 1.59256200 \\ \mathrm{H} & 0.43413900 & -2.20461900 & 1.16217200 \\ \mathrm{H} & -0.01561600 & -2.50065500 & -0.50723400 \\ \mathrm{H} & 2.14255200 & -0.52484700 & -1.69212800\end{array}$




$\begin{array}{lrrr}\mathrm{H} & 4.52066700 & -0.91421400 & -2.26716900 \\ \mathrm{H} & 5.80759700 & -2.68855000 & -1.10882400 \\ \mathrm{H} & 4.70196800 & -4.07088600 & 0.62827100 \\ \mathrm{H} & 2.33203600 & -3.67388600 & 1.20077900 \\ \mathrm{H} & -0.56384700 & 1.31700900 & -1.45437600 \\ \mathrm{~B} & -1.25916600 & -0.14167000 & 0.31364900 \\ \mathrm{O} & -1.92977100 & 0.76251100 & -0.60321200 \\ \mathrm{C} & -3.03404700 & 0.03534100 & -1.16901800 \\ \mathrm{C} & -3.38120400 & -0.95559200 & -0.01155500 \\ \mathrm{O} & -2.09056800 & -1.27543600 & 0.51415900 \\ \mathrm{C} & -2.53625700 & -0.68873200 & -2.42002600 \\ \mathrm{C} & -4.14281400 & 1.01185300 & -1.52142100 \\ \mathrm{C} & -4.05351000 & -2.24038500 & -0.46768800 \\ \mathrm{C} & -4.20853700 & -0.28713100 & 1.08806500 \\ \mathrm{H} & -1.75392700 & -1.40692500 & -2.16368400 \\ \mathrm{H} & -3.34829600 & -1.21358300 & -2.92861000 \\ \mathrm{H} & -2.11515000 & 0.04562900 & -3.11057900 \\ \mathrm{H} & -3.82095000 & 1.65396000 & -2.34396100 \\ \mathrm{H} & -5.03990300 & 0.47231700 & -1.83868300 \\ \mathrm{H} & -4.39278300 & 1.64413400 & -0.66989600 \\ \mathrm{H} & -3.39774800 & -2.81308900 & -1.12322900 \\ \mathrm{H} & -4.29023300 & -2.85646100 & 0.40222400 \\ \mathrm{H} & -4.98547700 & -2.02260100 & -0.99720400 \\ \mathrm{H} & -5.23898300 & -0.11752200 & 0.76682300 \\ \mathrm{H} & -4.21863900 & -0.94346400 & 1.96076900 \\ \mathrm{H} & -3.76938200 & 0.66879100 & 1.38184100 \\ \mathrm{C} & -0.94208600 & 0.81260200 & 1.77704400 \\ \mathrm{C} & -0.82983500 & 0.20751300 & 2.93743300 \\ \mathrm{C} & -0.69349900 & -0.47652600 & 4.03709500 \\ \mathrm{H} & -1.20277200 & 1.86880200 & 1.74868100 \\ \mathrm{H} & 0.24939700 & -0.50070500 & 4.57286200 \\ \mathrm{H} & -1.53204800 & -1.03064500 & 4.44498400\end{array}$

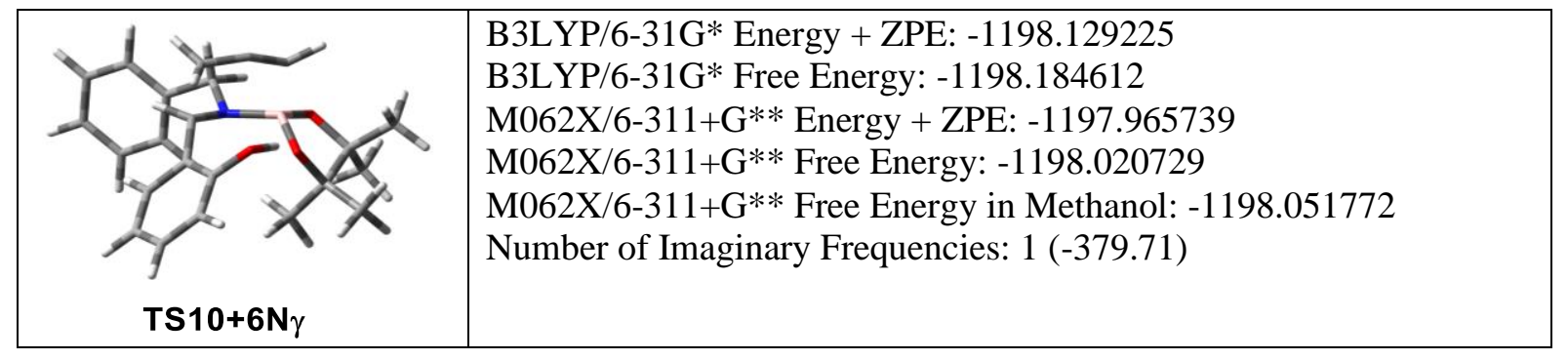

$\begin{array}{lrrr}\mathrm{C} & 1.00872800 & 3.64188900 & -1.53106000 \\ \mathrm{C} & 1.25282100 & 2.49807100 & -0.78563300 \\ \mathrm{C} & 0.33509100 & 2.01720200 & 0.15866900 \\ \mathrm{C} & -0.86416500 & 2.72443700 & 0.33918500 \\ \mathrm{C} & -1.09242500 & 3.89115000 & -0.39341800 \\ \mathrm{C} & -0.17074700 & 4.34969900 & -1.32070100 \\ \mathrm{C} & 0.83223500 & 0.80401200 & 0.89669300 \\ \mathrm{~N} & 0.46287700 & -0.44836800 & 0.61202300 \\ \mathrm{C} & 1.44308900 & -1.47950100 & 0.99840700\end{array}$




$\begin{array}{lrrr}\mathrm{C} & 2.75437900 & -1.32954700 & 0.26285300 \\ \mathrm{C} & 2.76782100 & -1.18162900 & -1.12609700 \\ \mathrm{C} & 3.96985000 & -1.05977900 & -1.81075100 \\ \mathrm{C} & 5.17739200 & -1.08409200 & -1.11523300 \\ \mathrm{C} & 5.17271800 & -1.22933300 & 0.26634200 \\ \mathrm{C} & 3.96474800 & -1.34746600 & 0.95064900 \\ \mathrm{O} & -1.81574800 & 2.35975400 & 1.23482200 \\ \mathrm{H} & 1.73795200 & 3.98406300 & -2.25483400 \\ \mathrm{H} & 2.18158000 & 1.95320200 & -0.93012000 \\ \mathrm{H} & -2.02335800 & 4.41467000 & -0.21295300 \\ \mathrm{H} & -0.37422700 & 5.25519800 & -1.88021100 \\ \mathrm{H} & 1.89571700 & 0.89986400 & 1.10789200 \\ \mathrm{H} & 1.61273400 & -1.44248800 & 2.08191700 \\ \mathrm{H} & 0.98810400 & -2.44424200 & 0.76743200 \\ \mathrm{H} & 1.82550800 & -1.15410000 & -1.66335900 \\ \mathrm{H} & 3.96733000 & -0.94691000 & -2.88865200 \\ \mathrm{H} & 6.11526500 & -0.98845400 & -1.64923100 \\ \mathrm{H} & 6.10691500 & -1.24506800 & 0.81526200 \\ \mathrm{H} & 3.96416400 & -1.45586200 & 2.03066900 \\ \mathrm{H} & -2.07897500 & 1.44059100 & 1.02018300 \\ \mathrm{~B} & -0.99679600 & -0.89775200 & 0.74253700 \\ \mathrm{O} & -1.24065800 & -2.21307500 & 0.25300600 \\ \mathrm{O} & -1.98983200 & -0.02819800 & 0.17354100 \\ \mathrm{C} & -2.51927500 & -0.69503500 & -0.98083900 \\ \mathrm{C} & -2.44618200 & -2.18572100 & -0.52043400 \\ \mathrm{C} & -1.59524500 & -0.39573300 & -2.16072600 \\ \mathrm{C} & -3.91878000 & -0.17188600 & -1.25322100 \\ \mathrm{C} & -2.32242800 & -3.18643200 & -1.65794900 \\ \mathrm{C} & -3.61277800 & -2.56005000 & 0.39383100 \\ \mathrm{H} & -0.60103800 & -0.81396500 & -1.98841800 \\ \mathrm{H} & -1.99326800 & -0.80113000 & -3.09383000 \\ \mathrm{H} & -1.49581500 & 0.68836500 & -2.25822800 \\ \mathrm{H} & -3.86273900 & 0.87100100 & -1.57367400 \\ \mathrm{H} & -1.93293600 & -1.46517400 & 2.94717900 \\ \mathrm{H} & -4.39568300 & -0.74940200 & -2.04988900 \\ \mathrm{H} & -4.53895100 & -0.22335900 & -0.35853400 \\ \mathrm{H} & -1.40815600 & -3.01762000 & -2.22663900 \\ \mathrm{H} & -2.29039900 & -4.19910000 & -1.25103300 \\ \mathrm{H} & -3.18033600 & -3.11449100 & -2.33278200 \\ \mathrm{H} & -4.54792500 & -2.65141600 & -0.16377600 \\ \mathrm{H} & -3.39250300 & -3.52004400 & 0.86478900 \\ \mathrm{H} & -3.74702500 & -1.80986200 & 1.17659700 \\ \mathrm{H} & -1.22125600 & -0.77013200 & 2.53054000 \\ \mathrm{H} & 0.56989400 & 0.21356100 & 2.97855900 \\ \mathrm{H} & & 2.22989400 & 2.88686900\end{array}$




\begin{tabular}{|l|l|} 
B3LYP/6-31G* Energy + ZPE: -1198.193191 \\
B3LYP/6-31G* Free Energy: -1198.252270 \\
M062X/6-311+G** Energy + ZPE: -1198.027008 \\
M062X/6-311+G** Free Energy: -1198.084214 \\
M062X/6-311+G** Free Energy in Methanol: -1198.114467 \\
Number of Imaginary Frequencies: 0
\end{tabular}

$\begin{array}{lrrr}\mathrm{C} & 2.30368800 & 1.33293100 & -2.54128700 \\ \mathrm{C} & 2.06225400 & 0.96117400 & -1.22711600 \\ \mathrm{C} & 0.95689900 & 1.43120400 & -0.50646200 \\ \mathrm{C} & 0.07367200 & 2.30093200 & -1.16752700 \\ \mathrm{C} & 0.32731200 & 2.69338700 & -2.48382600 \\ \mathrm{C} & 1.43198200 & 2.21706400 & -3.16998200 \\ \mathrm{C} & 0.85341100 & 0.99065100 & 0.95815200 \\ \mathrm{~N} & 0.15180700 & -0.29341700 & 1.14806200 \\ \mathrm{C} & 0.93649900 & -1.37063800 & 1.74388000 \\ \mathrm{C} & 2.03477900 & -1.85111300 & 0.82030800 \\ \mathrm{C} & 1.69642100 & -2.40233800 & -0.41708000 \\ \mathrm{C} & 2.68547300 & -2.77143400 & -1.31744100 \\ \mathrm{C} & 4.02966400 & -2.59472100 & -0.99030100 \\ \mathrm{C} & 4.37519900 & -2.05968100 & 0.24426700 \\ \mathrm{C} & 3.37795100 & -1.69088100 & 1.14629500 \\ \mathrm{O} & -1.04112700 & 2.82891900 & -0.58826300 \\ \mathrm{H} & 3.17285800 & 0.94783700 & -3.06027600 \\ \mathrm{H} & 2.76075300 & 0.29255200 & -0.73345000 \\ \mathrm{H} & -0.37605100 & 3.37747400 & -2.94372600 \\ \mathrm{H} & 1.61001000 & 2.53239500 & -4.19153100 \\ \mathrm{H} & 1.88683900 & 0.80510400 & 1.25898700 \\ \mathrm{H} & 1.36944300 & -1.02439000 & 2.68950400 \\ \mathrm{H} & 0.25396900 & -2.19187300 & 1.97169000 \\ \mathrm{H} & 0.64703100 & -2.51788200 & -0.67220300 \\ \mathrm{H} & 2.41286100 & -3.18998200 & -2.27923100 \\ \mathrm{H} & 4.80220800 & -2.87522500 & -1.69649900 \\ \mathrm{H} & 5.41794500 & -1.91986300 & 0.50406100 \\ \mathrm{H} & 3.64810500 & -1.25931500 & 2.10532400 \\ \mathrm{H} & -1.48492700 & 2.11983100 & -0.09332400 \\ \mathrm{~B} & -1.16254500 & -0.51424100 & 0.72668800 \\ \mathrm{O} & -2.01430400 & 0.50863200 & 0.31826400 \\ \mathrm{C} & -3.07412600 & -0.12486200 & -0.44101600 \\ \mathrm{C} & -3.14497900 & -1.52470400 & 0.25113700 \\ \mathrm{O} & -1.76908400 & -1.74971500 & 0.63075400 \\ \mathrm{C} & -2.59686200 & -0.19650700 & -1.88957000 \\ \mathrm{C} & -4.33160300 & 0.71632100 & -0.32551100 \\ \mathrm{C} & -3.59070400 & -2.65137300 & -0.66362100 \\ \mathrm{C} & -3.97618800 & -1.50374800 & 1.53012400 \\ \mathrm{H} & -1.68842400 & -0.79953700 & -1.97338300 \\ \mathrm{H} & -3.36408500 & -0.62031500 & -2.54063400 \\ \mathrm{H} & -2.36193300 & 0.81340300 & -2.23319400\end{array}$




$\begin{array}{lrrr}\mathrm{H} & -4.17780700 & 1.67279700 & -0.82962600 \\ \mathrm{H} & -5.17206200 & 0.20770600 & -0.80508900 \\ \mathrm{H} & -4.58430600 & 0.91149800 & 0.71631100 \\ \mathrm{H} & -2.90165600 & -2.77505700 & -1.49853300 \\ \mathrm{H} & -3.62228800 & -3.58668200 & -0.10206900 \\ \mathrm{H} & -4.59170700 & -2.45141400 & -1.05573400 \\ \mathrm{H} & -5.04133300 & -1.40381000 & 1.31056400 \\ \mathrm{H} & -3.81680000 & -2.44134600 & 2.06500600 \\ \mathrm{H} & -3.67247400 & -0.67951500 & 2.17934800 \\ \mathrm{C} & 0.32608100 & 2.03923100 & 1.92315800 \\ \mathrm{C} & 1.10123200 & 2.63158200 & 2.78689700 \\ \mathrm{C} & 1.89161600 & 3.22008000 & 3.64037300 \\ \mathrm{H} & -0.73333300 & 2.27390300 & 1.91467200 \\ \mathrm{H} & 2.38830600 & 4.15093800 & 3.39018600 \\ \mathrm{H} & 2.06703700 & 2.79783900 & 4.62383600\end{array}$

B3LYP/6-31G* Energy + ZPE: -1198.181729
B3LYP/6-31G* Free Energy: -1198.240946
M062X/6-311+G** Energy + ZPE: -1198.026046
M062X/6-311+G** Free Energy: -1198.081765
M062X/6-311+G** Free Energy in Methanol: -1198.118481
Number of Imaginary Frequencies: 0

$\begin{array}{lrrr}\mathrm{C} & 1.78382400 & -0.07548300 & 2.89596400 \\ \mathrm{C} & 0.94326100 & 0.39083900 & 1.89273400 \\ \mathrm{C} & 1.44440800 & 1.05743700 & 0.77680300 \\ \mathrm{C} & 2.81999900 & 1.28234000 & 0.70107800 \\ \mathrm{C} & 3.67371700 & 0.80887300 & 1.69598700 \\ \mathrm{C} & 3.15649300 & 0.12620800 & 2.78791100 \\ \mathrm{C} & 0.52188300 & 1.47379300 & -0.35926700 \\ \mathrm{~N} & -0.24826300 & 0.34690400 & -0.88439300 \\ \mathrm{~B} & -1.50167700 & -0.08072200 & -0.41300100 \\ \mathrm{O} & -2.16439300 & 0.44814200 & 0.68416000 \\ \mathrm{O} & -2.21046200 & -1.12173600 & -0.99122000 \\ \mathrm{O} & 3.29451700 & 1.97669000 & -0.37701100 \\ \mathrm{C} & -3.50590000 & -0.08132600 & 0.64990400 \\ \mathrm{C} & -3.29370500 & -1.43680800 & -0.09588100 \\ \mathrm{C} & -0.35562300 & 2.66468800 & 0.07842000 \\ \mathrm{C} & -1.27387300 & 3.09717500 & -0.97320800 \\ \mathrm{C} & -2.02277300 & 3.43494600 & -1.84901100 \\ \mathrm{C} & 0.41276800 & -0.38222000 & -1.96304200 \\ \mathrm{C} & 1.72429200 & -1.00970400 & -1.53014800 \\ \mathrm{C} & 1.74612500 & -1.91121900 & -0.46305600 \\ \mathrm{C} & 2.92213300 & -0.67359500 & -2.15061700 \\ \mathrm{C} & 4.12782900 & -1.22250800 & -1.71385600 \\ \mathrm{C} & 4.14022700 & -2.10782200 & -0.64400400 \\ \mathrm{C} & 2.94208900 & -2.45391800 & -0.01819300\end{array}$




\begin{tabular}{lrrr}
$\mathrm{C}$ & -4.35959700 & 0.89992600 & -0.14870500 \\
$\mathrm{C}$ & -4.02251500 & -0.21028700 & 2.07188100 \\
$\mathrm{C}$ & -4.48815000 & -1.90837300 & -0.90623300 \\
$\mathrm{C}$ & -2.80550300 & -2.54447700 & 0.83554300 \\
$\mathrm{H}$ & 1.37338600 & -0.59702900 & 3.75189300 \\
$\mathrm{H}$ & -0.12653900 & 0.21486800 & 1.95425300 \\
$\mathrm{H}$ & 4.74289000 & 0.97566600 & 1.60653700 \\
$\mathrm{H}$ & 3.82757900 & -0.24236000 & 3.55469600 \\
$\mathrm{H}$ & 1.14720400 & 1.82886400 & -1.18231700 \\
$\mathrm{H}$ & 4.24273700 & 1.83986200 & -0.45138000 \\
$\mathrm{H}$ & -0.92742400 & 2.40428300 & 0.97038900 \\
$\mathrm{H}$ & 0.31564500 & 3.48688400 & 0.34127900 \\
$\mathrm{H}$ & -2.68473100 & 3.74206700 & -2.62262900 \\
$\mathrm{H}$ & -0.27355400 & -1.15559600 & -2.31206800 \\
$\mathrm{H}$ & 0.60017900 & 0.29976600 & -2.80077600 \\
$\mathrm{H}$ & 0.81464500 & -2.15541600 & 0.03933400 \\
$\mathrm{H}$ & 2.91620900 & 0.03599500 & -2.97201100 \\
$\mathrm{H}$ & 5.05440500 & -0.95434300 & -2.20942700 \\
$\mathrm{H}$ & 5.07642800 & -2.52786600 & -0.29459200 \\
$\mathrm{H}$ & 2.94658000 & -3.13669700 & 0.82339600 \\
$\mathrm{H}$ & -4.00143000 & 0.97554800 & -1.17806300 \\
$\mathrm{H}$ & -5.40986200 & 0.59944600 & -0.15607700 \\
$\mathrm{H}$ & -4.27532500 & 1.88793100 & 0.30750100 \\
$\mathrm{H}$ & -4.14595400 & 0.78334300 & 2.50675400 \\
$\mathrm{H}$ & -4.99488300 & -0.71061900 & 2.07995600 \\
$\mathrm{H}$ & -3.33015900 & -0.77546700 & 2.69568000 \\
$\mathrm{H}$ & -4.73937700 & -1.19285600 & -1.68843800 \\
$\mathrm{H}$ & -4.25580000 & -2.86517300 & -1.37747800 \\
$\mathrm{H}$ & -5.35788300 & -2.04688300 & -0.25798100 \\
$\mathrm{H}$ & -3.59930400 & -2.88244100 & 1.50523400 \\
$\mathrm{H}$ & -2.47503700 & -3.39026400 & 0.22997400 \\
$\mathrm{H}$ & -1.96015500 & -2.20191700 & 1.43866000 \\
& & & \\
\hline & & & \\
& & & \\
& & &
\end{tabular}

B3LYP/6-31G* Energy + ZPE: -1198.156339
B3LYP/6-31G* Free Energy: -1198.216104
M062X/6-311+G** Energy + ZPE: -1197.977042
M062X/6-311+G** Free Energy: -1198.034012
M062X/6-311+G** Free Energy in Methanol: -1198.085574
Number of Imaginary Frequencies: 0

$\begin{array}{lrrr}\mathrm{C} & 1.42427000 & 3.55373800 & -0.18127000 \\ \mathrm{C} & 0.61483600 & 2.38830000 & -0.15438500 \\ \mathrm{C} & -0.64454300 & 2.37460300 & -0.83206400 \\ \mathrm{C} & -0.99645500 & 3.54225300 & -1.56682300 \\ \mathrm{C} & -0.18393700 & 4.64399200 & -1.58161500 \\ \mathrm{C} & 1.04187000 & 4.66718500 & -0.87486300 \\ \mathrm{C} & 1.03401400 & 1.18721800 & 0.46648700 \\ \mathrm{~N} & 2.10223600 & 1.01283200 & 1.19763500\end{array}$




\begin{tabular}{|c|c|c|c|}
\hline $\mathrm{C}$ & 2.49437200 & -0.32785800 & 1.67324000 \\
\hline $\mathrm{C}$ & 3.10392700 & -1.15444800 & 0.56681200 \\
\hline $\mathrm{C}$ & 4.48414200 & -1.22091400 & 0.39215700 \\
\hline $\mathrm{C}$ & 5.02251500 & -1.95292200 & -0.66167600 \\
\hline $\mathrm{C}$ & 4.18087000 & -2.62018700 & -1.54525400 \\
\hline $\mathrm{C}$ & 2.80103300 & -2.55973600 & -1.37094300 \\
\hline $\mathrm{C}$ & 2.26254200 & -1.83059600 & -0.31728900 \\
\hline $\mathrm{O}$ & -1.47536400 & 1.38489900 & -0.84221900 \\
\hline B & -1.89049400 & 0.33140100 & 0.25479000 \\
\hline $\mathrm{O}$ & -3.21158700 & -0.08136100 & -0.11904200 \\
\hline $\mathrm{O}$ & -1.04618500 & -0.86250900 & 0.18481900 \\
\hline $\mathrm{C}$ & -1.77828400 & -1.86196700 & -0.52911000 \\
\hline $\mathrm{C}$ & -3.24796100 & -1.50028500 & -0.15502800 \\
\hline $\mathrm{C}$ & -3.62177000 & -2.01403400 & 1.23895900 \\
\hline $\mathrm{C}$ & -4.28345800 & -1.94804100 & -1.17563700 \\
\hline $\mathrm{C}$ & -1.33645500 & -3.24275100 & -0.06510800 \\
\hline $\mathrm{C}$ & -1.50495800 & -1.69192600 & -2.02537400 \\
\hline $\mathrm{H}$ & 2.37811000 & 3.56048300 & 0.33694900 \\
\hline $\mathrm{H}$ & -1.94193800 & 3.52194000 & -2.09382500 \\
\hline $\mathrm{H}$ & -0.48770400 & 5.52062300 & -2.14321800 \\
\hline $\mathrm{H}$ & 1.66915400 & 5.54857000 & -0.89722300 \\
\hline $\mathrm{H}$ & 0.44924500 & 0.28429000 & 0.29687100 \\
\hline $\mathrm{H}$ & 2.71867400 & 1.79100000 & 1.39159700 \\
\hline $\mathrm{H}$ & 3.19315500 & -0.18939800 & 2.49862900 \\
\hline $\mathrm{H}$ & 1.58835800 & -0.79815800 & 2.06151500 \\
\hline $\mathrm{H}$ & 5.14238900 & -0.70543500 & 1.08476700 \\
\hline $\mathrm{H}$ & 6.09706200 & -2.00357600 & -0.79108000 \\
\hline $\mathrm{H}$ & 4.60007800 & -3.19095800 & -2.36527500 \\
\hline $\mathrm{H}$ & 2.14234600 & -3.08512200 & -2.05306600 \\
\hline $\mathrm{H}$ & 1.18591300 & -1.78430200 & -0.17395900 \\
\hline $\mathrm{H}$ & -2.85433700 & -1.73467900 & 1.96504900 \\
\hline $\mathrm{H}$ & -4.56098000 & -1.54387800 & 1.53663300 \\
\hline $\mathrm{H}$ & -3.75284800 & -3.09929900 & 1.25437100 \\
\hline $\mathrm{H}$ & -4.25000700 & -3.03268700 & -1.31634100 \\
\hline $\mathrm{H}$ & -5.28085700 & -1.67924400 & -0.82165700 \\
\hline $\mathrm{H}$ & -4.12080700 & -1.45807800 & -2.13554900 \\
\hline $\mathrm{H}$ & -1.31937700 & -3.29888400 & 1.02359400 \\
\hline $\mathrm{H}$ & -2.01610600 & -4.00942300 & -0.44859400 \\
\hline $\mathrm{H}$ & -0.33478000 & -3.47045800 & -0.43951400 \\
\hline $\mathrm{H}$ & -0.42412900 & -1.72546400 & -2.19063100 \\
\hline $\mathrm{H}$ & -1.96973800 & -2.48879400 & -2.61185100 \\
\hline $\mathrm{H}$ & -1.87425400 & -0.72550400 & -2.37422500 \\
\hline $\mathrm{C}$ & -1.79658900 & 1.10998000 & 1.67772500 \\
\hline $\mathrm{C}$ & -1.02099800 & 0.80297300 & 2.67564600 \\
\hline $\mathrm{C}$ & -0.23150800 & 0.47029400 & 3.66723000 \\
\hline $\mathrm{H}$ & -2.48172300 & 1.94629400 & 1.82604600 \\
\hline $\mathrm{H}$ & -0.53949300 & -0.25247700 & 4.41478600 \\
\hline $\mathrm{H}$ & 0.74164400 & 0.93832400 & 3.78843700 \\
\hline
\end{tabular}




B3LYP/6-31G* Energy + ZPE: -1198.136173
B3LYP/6-31G* Free Energy: -1198.192315
M062X/6-311+G** Energy + ZPE: -1197.958948
M062X/6-311+G** Free Energy: -1198.014959
M062X/6-311+G** Free Energy in Methanol: -1198.056902
Number of Imaginary Frequencies: 1 (-436.95)

$\begin{array}{lrrr}\mathrm{C} & -1.11651900 & 2.99894800 & 0.09808900 \\ \mathrm{C} & -0.17044800 & 1.97599900 & -0.11299200 \\ \mathrm{C} & 1.15396700 & 2.33247800 & -0.45123200 \\ \mathrm{C} & 1.47148200 & 3.69903800 & -0.58236100 \\ \mathrm{C} & 0.52871100 & 4.67793900 & -0.36108800 \\ \mathrm{C} & -0.78160400 & 4.33260000 & -0.01001600 \\ \mathrm{C} & -0.60554200 & 0.57290400 & 0.01225100 \\ \mathrm{~N} & -1.90338100 & 0.33343000 & -0.23290300 \\ \mathrm{C} & -2.47064000 & -0.99699800 & -0.14669900 \\ \mathrm{C} & -3.95639200 & -0.94662700 & 0.11896900 \\ \mathrm{C} & -4.45152100 & -0.20504000 & 1.19301300 \\ \mathrm{C} & -5.81625400 & -0.16212900 & 1.44680600 \\ \mathrm{C} & -6.70117200 & -0.86099400 & 0.62896300 \\ \mathrm{C} & -6.21467000 & -1.59968300 & -0.44232500 \\ \mathrm{C} & -4.84625300 & -1.63859900 & -0.69746300 \\ \mathrm{O} & 2.11500400 & 1.47189400 & -0.71081100 \\ \mathrm{~B} & 1.98651700 & 0.01351900 & -0.54557800 \\ \mathrm{O} & 3.14998700 & -0.61313100 & -1.07854600 \\ \mathrm{O} & 1.83399400 & -0.43794600 & 0.81749800 \\ \mathrm{C} & 3.13295800 & -0.85506000 & 1.24737200 \\ \mathrm{C} & 3.74005900 & -1.43417000 & -0.07374300 \\ \mathrm{C} & 3.32348100 & -2.88718000 & -0.30797700 \\ \mathrm{C} & 5.25522200 & -1.31704900 & -0.17217000 \\ \mathrm{C} & 2.98223900 & -1.86198700 & 2.37703900 \\ \mathrm{C} & 3.89760600 & 0.37419900 & 1.74617300 \\ \mathrm{H} & -2.12136200 & 2.72863600 & 0.40307300 \\ \mathrm{H} & 2.48959200 & 3.94142900 & -0.86078300 \\ \mathrm{H} & 0.80751100 & 5.72103800 & -0.45597100 \\ \mathrm{H} & -1.52159400 & 5.09791500 & 0.18593200 \\ \mathrm{H} & -0.13829800 & -0.06311500 & 0.75810300 \\ \mathrm{H} & -2.36699400 & 0.95887100 & -0.88143200 \\ \mathrm{H} & -2.27154900 & -1.53163000 & -1.08513500 \\ \mathrm{H} & -1.94579400 & -1.53002300 & 0.65162600 \\ \mathrm{H} & -3.76028100 & 0.34019400 & 1.82752700 \\ \mathrm{H} & -6.19088800 & 0.41545900 & 2.28352900 \\ \mathrm{H} & -7.76572800 & -0.82694600 & 0.82693200 \\ \mathrm{H} & -6.89793000 & -2.14247300 & -1.08445900 \\ \mathrm{H} & -4.46928900 & -2.21024900 & -1.53930900 \\ \mathrm{H} & 2.25292700 & -3.01411700 & -0.14495900 \\ \mathrm{H} & 3.55367100 & -3.14886500 & -1.34370400 \\ \mathrm{H} & 3.86413600 & -3.56957800 & 0.35323900 \\ \mathrm{H} & 5.74112400 & -1.83973200 & 0.65739700 \\ & & & \\ & & & \end{array}$




$\begin{array}{lrrr}\mathrm{H} & 5.59297000 & -1.76944100 & -1.10712500 \\ \mathrm{H} & 5.56684100 & -0.27310700 & -0.16858200 \\ \mathrm{H} & 2.30839300 & -2.67154900 & 2.09718800 \\ \mathrm{H} & 3.95386300 & -2.28667600 & 2.64561100 \\ \mathrm{H} & 2.57381600 & -1.36307300 & 3.25878600 \\ \mathrm{H} & 3.30682200 & 0.85663600 & 2.52798200 \\ \mathrm{H} & 4.86861900 & 0.09664100 & 2.16383000 \\ \mathrm{H} & 4.05037600 & 1.09273300 & 0.93888300 \\ \mathrm{C} & 0.52228400 & -0.34805700 & -1.46645200 \\ \mathrm{C} & 0.22563400 & -1.62364300 & -1.57436100 \\ \mathrm{C} & 0.03672500 & -2.91287600 & -1.64763700 \\ \mathrm{H} & 0.33240400 & 0.27531300 & -2.34183400 \\ \mathrm{H} & 0.84130600 & -3.54667600 & -2.00802500 \\ \mathrm{H} & -0.89159300 & -3.38985500 & -1.35819800\end{array}$

B3LYP/6-31G* Energy + ZPE: -1198.138407
B3LYP/6-31G* Free Energy: -1198.193604
M062X/6-311+G** Energy + ZPE: -1197.961318
M062X/6-311+G** Free Energy: -1198.016819
M062X/6-311+G** Free Energy in Methanol: -1198.058975
Number of Imaginary Frequencies: 1 (-436.95)

$\begin{array}{lrrr}\mathrm{C} & -2.74603200 & -4.06712500 & -1.08243700 \\ \mathrm{C} & -1.51618200 & -3.98434900 & -0.44156100 \\ \mathrm{C} & -0.89687100 & -2.75056500 & -0.25129700 \\ \mathrm{C} & -1.52223300 & -1.57933300 & -0.71330700 \\ \mathrm{C} & -2.76086400 & -1.66323000 & -1.34379000 \\ \mathrm{C} & -3.36644100 & -2.90141100 & -1.52391300 \\ \mathrm{C} & 0.37969400 & -2.67186100 & 0.50825200 \\ \mathrm{~N} & 1.30999000 & -1.77039600 & 0.13119800 \\ \mathrm{C} & 2.54227400 & -1.60405300 & 0.88450100 \\ \mathrm{C} & 3.46915100 & -0.64289000 & 0.18165200 \\ \mathrm{C} & 3.04769600 & 0.66734900 & -0.05894300 \\ \mathrm{C} & 3.89005600 & 1.55633300 & -0.71610000 \\ \mathrm{C} & 5.15870000 & 1.15275800 & -1.12528100 \\ \mathrm{C} & 5.58179800 & -0.14809500 & -0.88073600 \\ \mathrm{C} & 4.73585900 & -1.04374900 & -0.23277700 \\ \mathrm{O} & -0.88052900 & -0.40326100 & -0.57017000 \\ \mathrm{~B} & -1.26778700 & 0.62169800 & 0.42199200 \\ \mathrm{O} & -0.19989500 & 1.58188100 & 0.47943700 \\ \mathrm{O} & -2.48842500 & 1.32764700 & 0.15665400 \\ \mathrm{C} & -0.76982700 & 2.88590500 & 0.36844300 \\ \mathrm{C} & -2.11505100 & 2.59995400 & -0.37689100 \\ \mathrm{C} & 0.20137600 & 3.77823100 & -0.39128200 \\ \mathrm{C} & -0.99438700 & 3.44074800 & 1.77639100 \\ \mathrm{C} & -1.91439300 & 2.45537300 & -1.88766500 \\ \mathrm{C} & -3.22086700 & 3.60312600 & -0.08941600 \\ \mathrm{H} & -3.21790200 & -5.03029900 & -1.23092500\end{array}$




\begin{tabular}{lrrr}
$\mathrm{H}$ & -1.02956900 & -4.88594300 & -0.08122300 \\
$\mathrm{H}$ & -3.22664000 & -0.74219800 & -1.67223800 \\
$\mathrm{H}$ & -4.33109000 & -2.95763500 & -2.01484200 \\
$\mathrm{H}$ & 0.79531700 & -3.63652500 & 0.79727400 \\
$\mathrm{H}$ & 0.92480400 & -0.92318200 & -0.28436400 \\
$\mathrm{H}$ & 2.30570400 & -1.21311200 & 1.88602600 \\
$\mathrm{H}$ & 3.01943400 & -2.58049100 & 1.00938500 \\
$\mathrm{H}$ & 2.05979200 & 0.99691100 & 0.25960400 \\
$\mathrm{H}$ & 3.55386300 & 2.56909600 & -0.90587300 \\
$\mathrm{H}$ & 5.81357200 & 1.85026500 & -1.63398700 \\
$\mathrm{H}$ & 6.56625700 & -0.46985500 & -1.19913300 \\
$\mathrm{H}$ & 5.06409900 & -2.06204000 & -0.05175000 \\
$\mathrm{H}$ & 0.50148000 & 3.31340400 & -1.33076900 \\
$\mathrm{H}$ & -0.24797900 & 4.75290100 & -0.60305400 \\
$\mathrm{H}$ & 1.09607000 & 3.94117100 & 0.21521700 \\
$\mathrm{H}$ & -0.05203800 & 3.38959200 & 2.32550000 \\
$\mathrm{H}$ & -1.32949000 & 4.48068200 & 1.75154600 \\
$\mathrm{H}$ & -1.74042800 & 2.85078100 & 2.31266500 \\
$\mathrm{H}$ & -1.10498100 & 1.75496900 & -2.10358900 \\
$\mathrm{H}$ & -2.83549000 & 2.05860300 & -2.32062900 \\
$\mathrm{H}$ & -1.69595200 & 3.41644300 & -2.36017600 \\
$\mathrm{H}$ & -2.91336000 & 4.61217400 & -0.37952700 \\
$\mathrm{H}$ & -4.11098100 & 3.33822400 & -0.66392100 \\
$\mathrm{H}$ & -3.48630600 & 3.60313800 & 0.96776200 \\
$\mathrm{C}$ & -1.45377000 & -0.12033300 & 1.99648700 \\
$\mathrm{C}$ & -0.90093700 & -1.21581500 & 2.29797700 \\
$\mathrm{C}$ & -0.23914200 & -2.40643100 & 2.38138500 \\
$\mathrm{H}$ & -2.03851200 & 0.48851600 & 2.67656900 \\
$\mathrm{H}$ & -0.85015500 & -3.30148100 & 2.45935100 \\
$\mathrm{H}$ & 0.69262700 & -2.42318600 & 2.94122100 \\
& & & \\
\hline
\end{tabular}

B3LYP/6-31G* Energy + ZPE: -1198.201221
B3LYP/6-31G* Free Energy: -1198.260942
M062X/6-311+G** Energy + ZPE: -1198.025601
M062X/6-311+G** Free Energy: -1198.083974
M062X/6-311+G** Free Energy in Methanol: -1198.118752
Number of Imaginary Frequencies: 0

$\begin{array}{lrrr}\text { C } & 0.78666600 & 3.06289300 & -0.31005000 \\ \mathrm{C} & 0.01220300 & 2.04370100 & 0.24069600 \\ \mathrm{C} & -1.36270800 & 2.27034500 & 0.37362700 \\ \mathrm{C} & -1.93471400 & 3.47808300 & -0.00008700 \\ \mathrm{C} & -1.14025000 & 4.48695000 & -0.53103000 \\ \mathrm{C} & 0.22312000 & 4.27645500 & -0.69158000 \\ \mathrm{C} & 0.61910700 & 0.73155900 & 0.71105900 \\ \mathrm{~N} & 2.00605700 & 0.59547700 & 0.28299900 \\ \mathrm{C} & 2.52387300 & -0.76055900 & 0.44099300\end{array}$




\begin{tabular}{|c|c|c|c|}
\hline $\mathrm{C}$ & 3.95400000 & -0.85793500 & -0.03700300 \\
\hline $\mathrm{C}$ & 4.88680900 & -1.61666800 & 0.66727800 \\
\hline $\mathrm{C}$ & 6.19659700 & -1.73604100 & 0.21083500 \\
\hline $\mathrm{C}$ & 6.58873400 & -1.08778200 & -0.95453600 \\
\hline $\mathrm{C}$ & 5.66464300 & -0.32144500 & -1.66005100 \\
\hline $\mathrm{C}$ & 4.35619500 & -0.20772000 & -1.20527400 \\
\hline $\mathrm{O}$ & -2.18958500 & 1.31339800 & 0.92360100 \\
\hline B & -2.53922300 & 0.18969000 & 0.24445500 \\
\hline $\mathrm{O}$ & -3.41733200 & -0.72667600 & 0.75717600 \\
\hline $\mathrm{O}$ & -2.06728100 & -0.13810900 & -1.00120500 \\
\hline $\mathrm{C}$ & -2.85871900 & -1.25788900 & -1.45933300 \\
\hline $\mathrm{C}$ & -3.35367000 & -1.88181100 & -0.11004500 \\
\hline $\mathrm{C}$ & -2.34093500 & -2.84913100 & 0.49645700 \\
\hline $\mathrm{C}$ & -4.72741000 & -2.52461200 & -0.17731500 \\
\hline $\mathrm{C}$ & -1.98118300 & -2.16871700 & -2.29877100 \\
\hline $\mathrm{C}$ & -3.99856500 & -0.68692000 & -2.29732200 \\
\hline $\mathrm{H}$ & 1.84414200 & 2.89013800 & -0.46515000 \\
\hline $\mathrm{H}$ & -3.00193600 & 3.60766000 & 0.13379400 \\
\hline $\mathrm{H}$ & -1.58912200 & 5.42880600 & -0.82290900 \\
\hline $\mathrm{H}$ & 0.84996200 & 5.05103900 & -1.11629100 \\
\hline $\mathrm{H}$ & 0.06737100 & -0.08637100 & 0.23778400 \\
\hline $\mathrm{H}$ & 2.59726600 & 1.23735200 & 0.80666600 \\
\hline $\mathrm{H}$ & 2.45762400 & -1.12812700 & 1.47519400 \\
\hline $\mathrm{H}$ & 1.89286800 & -1.41790000 & -0.16964800 \\
\hline $\mathrm{H}$ & 4.58620500 & -2.11733200 & 1.58213200 \\
\hline $\mathrm{H}$ & 6.91053800 & -2.32960300 & 0.76986800 \\
\hline $\mathrm{H}$ & 7.60847800 & -1.17542100 & -1.31030100 \\
\hline $\mathrm{H}$ & 5.96466400 & 0.18824400 & -2.56840500 \\
\hline $\mathrm{H}$ & 3.63172900 & 0.38929800 & -1.74723200 \\
\hline $\mathrm{H}$ & -1.34709100 & -2.39769000 & 0.55382900 \\
\hline $\mathrm{H}$ & -2.65504900 & -3.09107100 & 1.51347100 \\
\hline $\mathrm{H}$ & -2.28129500 & -3.77337100 & -0.08244900 \\
\hline $\mathrm{H}$ & -4.72932700 & -3.34002000 & -0.90566200 \\
\hline $\mathrm{H}$ & -4.98483200 & -2.93644400 & 0.80009200 \\
\hline $\mathrm{H}$ & -5.49139500 & -1.79945600 & -0.45543400 \\
\hline $\mathrm{H}$ & -1.08117100 & -2.45910400 & -1.75747400 \\
\hline $\mathrm{H}$ & -2.52970000 & -3.07068500 & -2.58378100 \\
\hline $\mathrm{H}$ & -1.68158000 & -1.64598700 & -3.20869000 \\
\hline $\mathrm{H}$ & -3.57337800 & -0.09574000 & -3.10986700 \\
\hline $\mathrm{H}$ & -4.61245700 & -1.48241900 & -2.72469700 \\
\hline $\mathrm{H}$ & -4.63737100 & -0.03646300 & -1.69535200 \\
\hline $\mathrm{C}$ & 0.45770900 & 0.60878500 & 2.22260100 \\
\hline $\mathrm{C}$ & -0.26188000 & -0.31517500 & 2.79093900 \\
\hline $\mathrm{C}$ & -1.03952100 & -1.22757500 & 3.30266100 \\
\hline $\mathrm{H}$ & 0.96510600 & 1.35856700 & 2.82680000 \\
\hline $\mathrm{H}$ & -2.09593600 & -1.02445800 & 3.44737600 \\
\hline $\mathrm{H}$ & -0.65763100 & -2.20189200 & 3.58799200 \\
\hline
\end{tabular}




B3LYP/6-31G* Energy + ZPE: -1198.189570
B3LYP/6-31G* Free Energy: -1198.249792
M062X/6-311+G** Energy + ZPE: -1198.020342
M062X/6-311+G** Free Energy: -1198.078586
M062X/6-311+G** Free Energy in Methanol: -1198.114672
Number of Imaginary Frequencies: 0

$\begin{array}{lrrr}\mathrm{C} & 0.79306100 & 4.58314800 & -0.41765900 \\ \mathrm{C} & -0.14186300 & 3.88841700 & 0.34079100 \\ \mathrm{C} & -0.21248700 & 2.49595600 & 0.32242400 \\ \mathrm{C} & 0.69576300 & 1.81138100 & -0.48593400 \\ \mathrm{C} & 1.62623600 & 2.49229900 & -1.26220900 \\ \mathrm{C} & 1.67859100 & 3.87922800 & -1.22478100 \\ \mathrm{C} & -1.25136100 & 1.78187500 & 1.16310200 \\ \mathrm{~N} & -2.13836700 & 0.98478300 & 0.33320500 \\ \mathrm{C} & -3.29528300 & 0.46868500 & 1.05766100 \\ \mathrm{C} & -4.14335300 & -0.40290000 & 0.16300000 \\ \mathrm{C} & -4.55938500 & -1.66276200 & 0.58676100 \\ \mathrm{C} & -5.35618200 & -2.46055300 & -0.23091100 \\ \mathrm{C} & -5.73784400 & -2.00454100 & -1.48670000 \\ \mathrm{C} & -5.31723200 & -0.74968400 & -1.92192500 \\ \mathrm{C} & -4.52403800 & 0.04498000 & -1.10343000 \\ \mathrm{O} & 0.61500700 & 0.43809900 & -0.54719200 \\ \mathrm{~B} & 1.71111300 & -0.35212600 & -0.35199200 \\ \mathrm{O} & 1.72610300 & -1.67602900 & -0.69255500 \\ \mathrm{O} & 2.90590500 & 0.10245400 & 0.14324900 \\ \mathrm{C} & 2.99132000 & -2.20981600 & -0.23985200 \\ \mathrm{C} & 3.88148100 & -0.92274200 & -0.14585200 \\ \mathrm{C} & 3.47490800 & -3.24182100 & -1.24387600 \\ \mathrm{C} & 2.76124000 & -2.85829900 & 1.12119200 \\ \mathrm{C} & 4.52511500 & -0.55370500 & -1.47998500 \\ \mathrm{C} & 4.92137200 & -0.94187600 & 0.95952700 \\ \mathrm{H} & 0.82276000 & 5.66521200 & -0.38456500 \\ \mathrm{H} & -0.84321500 & 4.43777400 & 0.96006600 \\ \mathrm{H} & 2.30602200 & 1.92407600 & -1.88622100 \\ \mathrm{H} & 2.40763800 & 4.40587600 & -1.82862400 \\ \mathrm{H} & -1.87036500 & 2.56781100 & 1.60759100 \\ \mathrm{H} & -1.61760400 & 0.21374900 & -0.07862800 \\ \mathrm{H} & -3.02276700 & -0.10973000 & 1.95255300 \\ \mathrm{H} & -3.88462700 & 1.33110800 & 1.39478000 \\ \mathrm{H} & -4.25205200 & -2.02338200 & 1.56317900 \\ \mathrm{H} & -5.67280900 & -3.43879000 & 0.11175700 \\ \mathrm{H} & -6.35581500 & -2.62385500 & -2.12644400 \\ \mathrm{H} & -5.60830300 & -0.39180500 & -2.90273900 \\ \mathrm{H} & -4.18048300 & 1.01661400 & -1.44030600 \\ \mathrm{H} & 3.49660200 & -2.83579200 & -2.25453300 \\ \mathrm{H} & 4.47717200 & -3.58921300 & -0.97833600\end{array}$




$\begin{array}{lrrr}\mathrm{H} & 2.80126900 & -4.10045100 & -1.23555700 \\ \mathrm{H} & 2.00124500 & -3.63510400 & 1.01501000 \\ \mathrm{H} & 3.67762100 & -3.31974700 & 1.49532700 \\ \mathrm{H} & 2.40727100 & -2.12556500 & 1.84917800 \\ \mathrm{H} & 3.78288400 & -0.54551000 & -2.28243500 \\ \mathrm{H} & 4.95429300 & 0.44634600 & -1.39538600 \\ \mathrm{H} & 5.31887700 & -1.25397000 & -1.74769000 \\ \mathrm{H} & 5.62334300 & -1.76657800 & 0.80911800 \\ \mathrm{H} & 5.48346400 & -0.00645200 & 0.94467100 \\ \mathrm{H} & 4.45603200 & -1.04652300 & 1.93889700 \\ \mathrm{C} & -0.31450200 & -1.58497000 & 1.92091500 \\ \mathrm{C} & -0.40010400 & -0.40736000 & 2.14106600 \\ \mathrm{C} & -0.61174400 & 1.01709100 & 2.38170600 \\ \mathrm{H} & -0.22712800 & -2.62308500 & 1.70744200 \\ \mathrm{H} & 0.33077400 & 1.49735700 & 2.65616900 \\ \mathrm{H} & -1.27745800 & 1.10839200 & 3.24504600\end{array}$

B3LYP/6-31G* Energy + ZPE: -1237.423811
B3LYP/6-31G* Free Energy: -1237.484527
M062X/6-311+G** Energy + ZPE: -1237.249303
M062X/6-311+G** Free Energy: -1237.306669
M062X/6-311+G** Free Energy in Methanol: -1237.342233
Number of Imaginary Frequencies: 0

$\begin{array}{lrrr}\mathrm{C} & -3.95909900 & 2.26062000 & 0.45394400 \\ \mathrm{C} & -2.56643400 & 2.27400800 & 0.41229100 \\ \mathrm{C} & -1.84702700 & 1.17452600 & 0.86041900 \\ \mathrm{C} & -2.51482900 & 0.05011700 & 1.36134000 \\ \mathrm{C} & -3.90574200 & 0.03152500 & 1.40346000 \\ \mathrm{C} & -4.61581800 & 1.14000500 & 0.94599100 \\ \mathrm{C} & -0.36871200 & 1.12688200 & 0.79809600 \\ \mathrm{~N} & 0.29784400 & 0.93784200 & -0.26638500 \\ \mathrm{C} & -0.34932600 & 0.72052100 & -1.57806200 \\ \mathrm{C} & -1.05432900 & -0.61637400 & -1.64178500 \\ \mathrm{C} & -2.44337400 & -0.69888700 & -1.70427700 \\ \mathrm{C} & -3.07170100 & -1.94055100 & -1.75499700 \\ \mathrm{C} & -2.31367600 & -3.10693400 & -1.73846100 \\ \mathrm{C} & -0.92386000 & -3.02841200 & -1.67872600 \\ \mathrm{C} & -0.29670200 & -1.78900600 & -1.63706000 \\ \mathrm{O} & -1.70867500 & -0.96828000 & 1.73661500 \\ \mathrm{C} & -2.30380300 & -2.22129800 & 2.02391400 \\ \mathrm{~B} & 1.94247600 & 1.02959000 & -0.24637300 \\ \mathrm{O} & 2.40764600 & 0.87732500 & 1.10713600 \\ \mathrm{O} & 2.42294900 & -0.11594800 & -0.99834000 \\ \mathrm{C} & 3.39547900 & -0.77836500 & -0.19075200 \\ \mathrm{C} & 2.90525100 & -0.45365600 & 1.25542700 \\ \mathrm{C} & 4.00229500 & -0.45059200 & 2.30923900 \\ \mathrm{C} & 1.76483000 & -1.37717800 & 1.69678000 \\ \mathrm{C} & 4.76447000 & -0.15945000 & -0.48235800 \\ & & & \end{array}$




\begin{tabular}{lrrr}
$\mathrm{C}$ & 3.41585700 & -2.26081600 & -0.53461000 \\
$\mathrm{C}$ & 2.25977000 & 2.48422600 & -0.89572400 \\
$\mathrm{C}$ & 1.38549000 & 3.43078800 & -1.07059900 \\
$\mathrm{C}$ & 0.45082800 & 4.33629900 & -1.22951800 \\
$\mathrm{H}$ & -4.52174300 & 3.11766900 & 0.10655600 \\
$\mathrm{H}$ & -2.02768500 & 3.13286100 & 0.02460600 \\
$\mathrm{H}$ & -4.43668900 & -0.83614800 & 1.77128000 \\
$\mathrm{H}$ & -5.69878200 & 1.12007300 & 0.97839100 \\
$\mathrm{H}$ & 0.22399000 & 1.24232700 & 1.70525400 \\
$\mathrm{H}$ & -1.04205000 & 1.54101300 & -1.77168100 \\
$\mathrm{H}$ & 0.45393500 & 0.76401700 & -2.31339900 \\
$\mathrm{H}$ & -3.03910100 & 0.20950800 & -1.70045000 \\
$\mathrm{H}$ & -4.15344400 & -1.99319500 & -1.80564800 \\
$\mathrm{H}$ & -2.80290400 & -4.07342800 & -1.78103400 \\
$\mathrm{H}$ & -0.32788100 & -3.93413700 & -1.67774100 \\
$\mathrm{H}$ & 0.78464600 & -1.70282100 & -1.60506800 \\
$\mathrm{H}$ & -1.47867900 & -2.90800800 & 2.19957200 \\
$\mathrm{H}$ & -2.92952500 & -2.16437900 & 2.91968500 \\
$\mathrm{H}$ & -2.89332900 & -2.56669300 & 1.16995000 \\
$\mathrm{H}$ & 4.74339600 & 0.31960000 & 2.09711000 \\
$\mathrm{H}$ & 3.56731700 & -0.24409400 & 3.28970700 \\
$\mathrm{H}$ & 4.50100300 & -1.42342500 & 2.35412800 \\
$\mathrm{H}$ & 2.13514800 & -2.37990700 & 1.92640100 \\
$\mathrm{H}$ & 1.30851700 & -0.96562600 & 2.60073400 \\
$\mathrm{H}$ & 0.98831600 & -1.45760600 & 0.93120400 \\
$\mathrm{H}$ & 4.78083500 & 0.89093400 & -0.18284000 \\
$\mathrm{H}$ & 5.56733500 & -0.68502600 & 0.04068400 \\
$\mathrm{H}$ & 4.94965500 & -0.21601000 & -1.55681600 \\
$\mathrm{H}$ & 3.74185900 & -2.38960400 & -1.56930900 \\
$\mathrm{H}$ & 4.11605800 & -2.79578200 & 0.11355300 \\
$\mathrm{H}$ & 2.42946400 & -2.71342400 & -0.42465200 \\
$\mathrm{H}$ & 3.28821900 & 2.68651500 & -1.19590300 \\
$\mathrm{H}$ & 0.24147200 & 5.06557100 & -0.45404400 \\
$\mathrm{H}$ & -0.10101900 & 4.41361400 & -2.16079200 \\
& & & \\
\hline & & & \\
& & &
\end{tabular}

B3LYP/6-31G* Energy + ZPE: -1237.369399
B3LYP/6-31G* Free Energy: -1237.429372
M062X/6-311+G** Energy + ZPE: -1237.179632
M062X/6-311+G** Free Energy: -1237.237320
M062X/6-311+G** Free Energy in Methanol: -1237.275882
Number of Imaginary Frequencies: 1 (-522.34)

$\begin{array}{llrr}\mathrm{B} & 0.69950400 & -0.90874000 & -0.53511000 \\ \mathrm{O} & 1.88197000 & -1.02416400 & -1.32918400 \\ \mathrm{O} & 0.95716300 & -1.51841600 & 0.74489800 \\ \mathrm{C} & 2.11846300 & -2.33425100 & 0.59389100 \\ \mathrm{C} & 2.92038500 & -1.56361300 & -0.50681700 \\ \mathrm{C} & 3.82681500 & -2.44021400 & -1.35853200\end{array}$




\begin{tabular}{|c|c|c|c|}
\hline $\mathrm{C}$ & 3.72237300 & -0.40207400 & 0.08080900 \\
\hline $\mathrm{C}$ & 1.69639700 & -3.73121300 & 0.12936300 \\
\hline $\mathrm{C}$ & 2.82550200 & -2.43321400 & 1.93742700 \\
\hline $\mathrm{C}$ & -3.36782800 & -0.21986500 & 2.32758200 \\
\hline $\mathrm{C}$ & -2.27754300 & -0.22765800 & 1.46909800 \\
\hline $\mathrm{C}$ & -2.41126200 & 0.17396900 & 0.13831300 \\
\hline $\mathrm{C}$ & -3.67617200 & 0.55373900 & -0.34701400 \\
\hline $\mathrm{C}$ & -4.77244200 & 0.56859900 & 0.51562400 \\
\hline $\mathrm{C}$ & -4.60642000 & 0.18728200 & 1.84415400 \\
\hline $\mathrm{C}$ & -1.24753100 & 0.11876400 & -0.76351700 \\
\hline $\mathrm{N}$ & 0.01173800 & 0.45036100 & -0.34907000 \\
\hline $\mathrm{C}$ & 0.29084900 & 1.33748100 & 0.76451900 \\
\hline $\mathrm{C}$ & 1.25762200 & 2.44955300 & 0.39465000 \\
\hline $\mathrm{C}$ & 1.44337300 & 3.49765800 & 1.29777500 \\
\hline $\mathrm{C}$ & 2.33313500 & 4.52742900 & 1.01952200 \\
\hline $\mathrm{C}$ & 3.05293000 & 4.52054900 & -0.17248400 \\
\hline $\mathrm{C}$ & 2.87517700 & 3.47731000 & -1.07372800 \\
\hline $\mathrm{C}$ & 1.98214200 & 2.44489500 & -0.79423800 \\
\hline $\mathrm{O}$ & -3.73882300 & 0.89544500 & -1.65442000 \\
\hline $\mathrm{C}$ & -4.99265200 & 1.27776400 & -2.18912200 \\
\hline $\mathrm{H}$ & 3.25255500 & -3.18917600 & -1.90365600 \\
\hline $\mathrm{H}$ & 4.35327400 & -1.81849600 & -2.08571500 \\
\hline $\mathrm{H}$ & 4.57239000 & -2.94589000 & -0.73761100 \\
\hline $\mathrm{H}$ & 4.58495400 & -0.75681000 & 0.65077000 \\
\hline $\mathrm{H}$ & 4.07817500 & 0.22939600 & -0.73570200 \\
\hline $\mathrm{H}$ & 3.09838900 & 0.21430700 & 0.73260500 \\
\hline $\mathrm{H}$ & 1.26133000 & -3.70052900 & -0.87092700 \\
\hline $\mathrm{H}$ & 2.54515400 & -4.41972000 & 0.12081000 \\
\hline $\mathrm{H}$ & 0.94407200 & -4.11319900 & 0.82355200 \\
\hline $\mathrm{H}$ & 2.21587100 & -3.01973700 & 2.62857600 \\
\hline $\mathrm{H}$ & 3.79473900 & -2.92881800 & 1.82935700 \\
\hline $\mathrm{H}$ & 2.98064000 & -1.44500500 & 2.37056600 \\
\hline $\mathrm{H}$ & -3.25635800 & -0.53611400 & 3.35654400 \\
\hline $\mathrm{H}$ & -1.30397300 & -0.57735600 & 1.80127000 \\
\hline $\mathrm{H}$ & -5.74917000 & 0.87403600 & 0.16732700 \\
\hline $\mathrm{H}$ & -5.46615700 & 0.20402800 & 2.50405500 \\
\hline $\mathrm{H}$ & -1.48824200 & 0.23162600 & -1.81770400 \\
\hline $\mathrm{H}$ & -0.63440300 & 1.79477400 & 1.12455400 \\
\hline $\mathrm{H}$ & 0.71824600 & 0.75940400 & 1.59446600 \\
\hline $\mathrm{H}$ & 0.88147200 & 3.50664000 & 2.22740800 \\
\hline $\mathrm{H}$ & 2.46303000 & 5.33591900 & 1.72967800 \\
\hline $\mathrm{H}$ & 3.74717900 & 5.32253100 & -0.39426700 \\
\hline $\mathrm{H}$ & 3.43678100 & 3.46217700 & -2.00102100 \\
\hline $\mathrm{H}$ & 1.85570300 & 1.62121800 & -1.48805100 \\
\hline $\mathrm{H}$ & -4.81567400 & 1.48990700 & -3.24063600 \\
\hline $\mathrm{H}$ & -5.72268800 & 0.46862300 & -2.09638300 \\
\hline $\mathrm{H}$ & -5.37119500 & 2.17645900 & -1.69386800 \\
\hline $\mathrm{C}$ & -0.66167700 & -1.71695300 & -1.34292900 \\
\hline $\mathrm{C}$ & -1.42532200 & -2.55688600 & -0.68579100 \\
\hline $\mathrm{C}$ & -2.18735300 & -3.29414900 & 0.06836600 \\
\hline $\mathrm{H}$ & -0.64107200 & -1.75926200 & -2.43050600 \\
\hline
\end{tabular}




$$
\begin{array}{llll}
\mathrm{H} & -3.20590300 & -2.98080900 & 0.28321800 \\
\mathrm{H} & -1.82504600 & -4.22126800 & 0.49845600
\end{array}
$$

B3LYP/6-31G* Energy + ZPE: -1237.397867
B3LYP/6-31G* Free Energy: -1237.454723
M062X/6-311+G** Energy + ZPE: -1237.220518
M062X/6-311+G** Free Energy: -1237.274908
Mumber-311+G** Free Energy in Methanol: -1237.308703
Number of Imaginary Frequencies: 1 (-460.48)

$\begin{array}{lrrr}\mathrm{C} & -1.99977300 & -2.12225100 & -1.36196300 \\ \mathrm{C} & -1.06322800 & -2.84892600 & -0.94478900 \\ \mathrm{C} & 0.09019100 & -3.10194100 & -0.26993200 \\ \mathrm{H} & -2.83082100 & -2.20986700 & -2.04051800 \\ \mathrm{H} & 0.05043200 & -3.69880900 & 0.63635600 \\ \mathrm{H} & 1.01308500 & -3.15147700 & -0.83590600 \\ \mathrm{C} & 3.04663300 & -0.53972300 & 2.83792100 \\ \mathrm{C} & 1.78586900 & -0.81699300 & 2.32263900 \\ \mathrm{C} & 1.58395300 & -1.07040100 & 0.96709800 \\ \mathrm{C} & 2.70771400 & -1.11461900 & 0.12246400 \\ \mathrm{C} & 3.97232800 & -0.82357100 & 0.62919000 \\ \mathrm{C} & 4.13481600 & -0.52690200 & 1.97917200 \\ \mathrm{C} & 0.15725200 & -1.18622200 & 0.54170700 \\ \mathrm{~N} & -0.46718500 & -0.30607600 & -0.25938600 \\ \mathrm{C} & 0.20034500 & 0.40717900 & -1.34921700 \\ \mathrm{C} & 1.31457500 & 1.33819700 & -0.91974400 \\ \mathrm{C} & 1.35956600 & 1.92079000 & 0.34533400 \\ \mathrm{C} & 2.43010200 & 2.72869100 & 0.71414500 \\ \mathrm{C} & 3.45959900 & 2.98567100 & -0.18585100 \\ \mathrm{C} & 3.40356200 & 2.43935900 & -1.46410200 \\ \mathrm{C} & 2.33810000 & 1.62048900 & -1.82306400 \\ \mathrm{O} & 2.48693100 & -1.43870100 & -1.17737700 \\ \mathrm{C} & 3.59885300 & -1.49443000 & -2.05169700 \\ \mathrm{~B} & -1.96588900 & -0.55034600 & -0.41690400 \\ \mathrm{O} & -2.67735200 & -0.80401400 & 0.80182300 \\ \mathrm{O} & -2.65519100 & 0.46381700 & -1.14860400 \\ \mathrm{C} & -3.88896300 & 0.71758100 & -0.47040200 \\ \mathrm{C} & -3.54430500 & 0.31585000 & 0.99856200 \\ \mathrm{C} & -4.73616800 & -0.11957200 & 1.83510800 \\ \mathrm{C} & -2.76084000 & 1.41541000 & 1.71981600 \\ \mathrm{C} & -4.96878300 & -0.18396400 & -1.07129200 \\ \mathrm{C} & -4.26085600 & 2.17975300 & -0.65984400 \\ \mathrm{H} & 3.17072800 & -0.32620700 & 3.89191400 \\ \mathrm{H} & 0.92005900 & -0.79882500 & 2.97649100 \\ \mathrm{H} & 4.83623700 & -0.81927100 & -0.02040800 \\ \mathrm{H} & 5.12490900 & -0.29470900 & 2.35304100 \\ \mathrm{H} & -0.49200600 & -1.48554100 & 1.36065700 \\ \mathrm{H} & 0.59420900 & -0.30756300 & -2.07808100 \\ \mathrm{H} & -0.58260300 & 0.98790900 & -1.84167200\end{array}$




$\begin{array}{lrrr}\mathrm{H} & 0.57015300 & 1.70701300 & 1.05757900 \\ \mathrm{H} & 2.46334100 & 3.15201700 & 1.71141400 \\ \mathrm{H} & 4.29695300 & 3.60883200 & 0.10521400 \\ \mathrm{H} & 4.19313900 & 2.64345500 & -2.17886700 \\ \mathrm{H} & 2.30507100 & 1.17800500 & -2.81469500 \\ \mathrm{H} & 3.19762200 & -1.76719200 & -3.02600000 \\ \mathrm{H} & 4.09397200 & -0.52109800 & -2.11829800 \\ \mathrm{H} & 4.31473700 & -2.25495200 & -1.72543700 \\ \mathrm{H} & -5.20614900 & -1.00659700 & 1.41119200 \\ \mathrm{H} & -4.40458400 & -0.36040900 & 2.84742300 \\ \mathrm{H} & -5.47732600 & 0.68231500 & 1.89862300 \\ \mathrm{H} & -3.39696500 & 2.26765800 & 1.97066200 \\ \mathrm{H} & -2.35234800 & 1.00120000 & 2.64454700 \\ \mathrm{H} & -1.93168400 & 1.76606700 & 1.10038900 \\ \mathrm{H} & -4.75313100 & -1.23529300 & -0.86810100 \\ \mathrm{H} & -5.95748700 & 0.05090500 & -0.66964600 \\ \mathrm{H} & -4.98710700 & -0.03136700 & -2.15250100 \\ \mathrm{H} & -4.50187800 & 2.36191900 & -1.70936100 \\ \mathrm{H} & -5.13649500 & 2.43882100 & -0.05739300 \\ \mathrm{H} & -3.43267300 & 2.83177700 & -0.38268900\end{array}$

B3LYP/6-31G* Energy + ZPE: -1237.464618
B3LYP/6-31G* Free Energy: -1237.525015
M062X/6-311+G** Energy + ZPE: -1237.284507
M062X/6-311+G** Free Energy: -1237.343880
M062X/6-311+G** Free Energy in Methanol: -1237.343875
Number of Imaginary Frequencies: 0

$\begin{array}{lrrr}\mathrm{C} & -4.79328900 & -0.36088600 & 0.27816600 \\ \mathrm{C} & -3.52277500 & -0.01250100 & 0.73715400 \\ \mathrm{C} & -2.43130700 & -0.85070700 & 0.56007000 \\ \mathrm{C} & -2.62892400 & -2.07963100 & -0.09583700 \\ \mathrm{C} & -3.89319900 & -2.43871500 & -0.55753800 \\ \mathrm{C} & -4.96990200 & -1.57295500 & -0.36876300 \\ \mathrm{C} & -1.02851500 & -0.48643000 & 1.01448300 \\ \mathrm{~N} & -0.16293400 & -0.14773900 & -0.11538200 \\ \mathrm{O} & -1.52597000 & -2.85925400 & -0.23296500 \\ \mathrm{C} & -1.67210200 & -4.11600100 & -0.85892400 \\ \mathrm{C} & -0.65458700 & 0.66050200 & -1.23027000 \\ \mathrm{C} & -0.52770200 & 2.15979300 & -1.03847200 \\ \mathrm{C} & 0.70445600 & 2.78664300 & -1.23588900 \\ \mathrm{C} & 0.83664100 & 4.15866500 & -1.05643900 \\ \mathrm{C} & -0.26237300 & 4.92321800 & -0.67069800 \\ \mathrm{C} & -1.49320700 & 4.30634200 & -0.47021000 \\ \mathrm{C} & -1.62257900 & 2.93247400 & -0.65864500 \\ \mathrm{~B} & 1.18377800 & -0.54090200 & -0.07687500 \\ \mathrm{O} & 2.10780100 & -0.21581400 & -1.05851500 \\ \mathrm{O} & 1.74004800 & -1.29897100 & 0.93757000\end{array}$




$\begin{array}{lrrr}\mathrm{C} & 3.16715100 & -1.22284100 & 0.76340400 \\ \mathrm{C} & 3.29570800 & -0.98104900 & -0.77561900 \\ \mathrm{C} & 3.20808600 & -2.27657500 & -1.57897800 \\ \mathrm{C} & 4.51393900 & -0.17568200 & -1.19303400 \\ \mathrm{C} & 3.80066200 & -2.51023800 & 1.25980000 \\ \mathrm{C} & 3.65663700 & -0.02902600 & 1.58144000 \\ \mathrm{C} & -1.03905100 & 0.55172400 & 2.12512700 \\ \mathrm{C} & -0.24610000 & 1.58338400 & 2.18308500 \\ \mathrm{C} & 0.53961300 & 2.62139700 & 2.21603900 \\ \mathrm{H} & -5.62943500 & 0.31054800 & 0.42788100 \\ \mathrm{H} & -3.37127400 & 0.93565900 & 1.24194900 \\ \mathrm{H} & -4.05240200 & -3.38336100 & -1.05960100 \\ \mathrm{H} & -5.94940000 & -1.86176500 & -0.73130400 \\ \mathrm{H} & -0.60007400 & -1.39520500 & 1.45019100 \\ \mathrm{H} & -2.01725600 & -4.00550500 & -1.89165900 \\ \mathrm{H} & -0.68367000 & -4.57031900 & -0.85401500 \\ \mathrm{H} & -2.36890300 & -4.75466600 & -0.30670000 \\ \mathrm{H} & -0.07597600 & 0.37363500 & -2.11109900 \\ \mathrm{H} & -1.69858500 & 0.40589700 & -1.42935600 \\ \mathrm{H} & 1.55934900 & 2.18363500 & -1.52562700 \\ \mathrm{H} & 1.79705300 & 4.63498600 & -1.21873200 \\ \mathrm{H} & -0.16049200 & 5.99341100 & -0.53192700 \\ \mathrm{H} & -2.35398000 & 4.89532000 & -0.17434200 \\ \mathrm{H} & -2.58513400 & 2.45291000 & -0.51052700 \\ \mathrm{H} & 2.34253700 & -2.86661700 & -1.26807300 \\ \mathrm{H} & 3.09283200 & -2.02659600 & -2.63507300 \\ \mathrm{H} & 4.10960000 & -2.88137300 & -1.45870500 \\ \mathrm{H} & 5.43236000 & -0.68666100 & -0.89099600 \\ \mathrm{H} & 4.52448200 & -0.06565600 & -2.27917200 \\ \mathrm{H} & 4.49981600 & 0.81896100 & -0.74769700 \\ \mathrm{H} & 3.33467900 & -3.38147700 & 0.80030000 \\ \mathrm{H} & 4.87121200 & -2.52030400 & 1.03647800 \\ \mathrm{H} & 3.67461500 & -2.58476500 & 2.34154700 \\ \mathrm{H} & 3.33432300 & -0.16192400 & 2.61581800 \\ \mathrm{H} & 4.74556600 & 0.05460700 & 1.55848000 \\ \mathrm{H} & 3.22111900 & 0.90050800 & 1.20471800 \\ \mathrm{H} & -1.72228900 & 0.36510600 & 2.95107600 \\ \mathrm{H} & 0.22743700 & 3.56396900 & 1.77630000 \\ \mathrm{H} & 1.52482400 & 2.56261700 & 2.66648700\end{array}$

B3LYP/6-31G* Energy + ZPE: -1237.454112
B3LYP/6-31G* Free Energy: -1237.514230
M062X/6-311+G** Energy + ZPE: -1237.278241
M062X/6-311+G** Free Energy: -1237.336736
M062X/6-311+G** Free Energy in Methanol: -1237.368093
Number of Imaginary Frequencies: 0
C $\quad-2.10769600$
$0.18450400-2.61057800$
C
$-1.49083600$
$0.25554500-1.36154000$ 


\begin{tabular}{|c|c|c|c|}
\hline $\mathrm{C}$ & -1.61629400 & -0.76536500 & -0.43075800 \\
\hline $\mathrm{C}$ & -2.37067400 & -1.89814900 & -0.78922400 \\
\hline $\mathrm{C}$ & -2.98597700 & -1.98350800 & -2.03470500 \\
\hline $\mathrm{C}$ & -2.85442000 & -0.93349500 & -2.94210100 \\
\hline $\mathrm{C}$ & -0.95834000 & -0.73888900 & 0.94493200 \\
\hline $\mathrm{N}$ & 0.15731900 & 0.20360500 & 1.07016800 \\
\hline $\mathrm{C}$ & -2.03789500 & -0.54298700 & 2.02813500 \\
\hline $\mathrm{C}$ & -1.52366400 & -0.72375500 & 3.38484800 \\
\hline $\mathrm{C}$ & -1.08612800 & -0.87757400 & 4.49232300 \\
\hline $\mathrm{O}$ & -2.44488200 & -2.88324600 & 0.15305000 \\
\hline $\mathrm{C}$ & -3.10075500 & -4.08482800 & -0.19313300 \\
\hline $\mathrm{C}$ & 0.03208300 & 1.51032900 & 1.72387100 \\
\hline $\mathrm{C}$ & -0.46743300 & 2.61855800 & 0.81946100 \\
\hline $\mathrm{C}$ & 0.37878900 & 3.13235400 & -0.16678300 \\
\hline $\mathrm{C}$ & -0.07566500 & 4.09773400 & -1.05636600 \\
\hline $\mathrm{C}$ & -1.38462200 & 4.56860200 & -0.96729900 \\
\hline $\mathrm{C}$ & -2.22583400 & 4.07991000 & 0.02490700 \\
\hline $\mathrm{C}$ & -1.76537800 & 3.11210300 & 0.91525800 \\
\hline B & 1.39271600 & -0.17398800 & 0.51322700 \\
\hline $\mathrm{O}$ & 1.58911300 & -1.36387700 & -0.15931200 \\
\hline $\mathrm{O}$ & 2.54118700 & 0.59896900 & 0.56982000 \\
\hline $\mathrm{C}$ & 3.47963000 & -0.00014300 & -0.34676100 \\
\hline $\mathrm{C}$ & 3.00757900 & -1.49140400 & -0.37551900 \\
\hline $\mathrm{C}$ & 3.57371800 & -2.30634700 & 0.78460100 \\
\hline $\mathrm{C}$ & 3.24347200 & -2.20485800 & -1.69527400 \\
\hline $\mathrm{C}$ & 4.88999500 & 0.21026000 & 0.17480400 \\
\hline $\mathrm{C}$ & 3.29628800 & 0.69559900 & -1.69423800 \\
\hline $\mathrm{H}$ & -1.99570500 & 1.00065900 & -3.31370000 \\
\hline $\mathrm{H}$ & -0.89233000 & 1.12164100 & -1.11384900 \\
\hline $\mathrm{H}$ & -3.56211600 & -2.85637600 & -2.31047600 \\
\hline $\mathrm{H}$ & -3.33734400 & -1.00582300 & -3.90958700 \\
\hline $\mathrm{H}$ & -0.54091200 & -1.73668100 & 1.10339700 \\
\hline $\mathrm{H}$ & -2.49734900 & 0.44400700 & 1.92554200 \\
\hline $\mathrm{H}$ & -2.82102200 & -1.28092500 & 1.84707300 \\
\hline $\mathrm{H}$ & -0.70111400 & -1.01539000 & 5.47418100 \\
\hline $\mathrm{H}$ & -3.00779200 & -4.73913600 & 0.67104800 \\
\hline $\mathrm{H}$ & -4.16037800 & -3.91081200 & -0.40469100 \\
\hline $\mathrm{H}$ & -2.62785700 & -4.55493700 & -1.06082700 \\
\hline $\mathrm{H}$ & -0.60470000 & 1.43186500 & 2.60632500 \\
\hline $\mathrm{H}$ & 1.02873800 & 1.77569500 & 2.08247500 \\
\hline $\mathrm{H}$ & 1.39370400 & 2.75239100 & -0.23428100 \\
\hline $\mathrm{H}$ & 0.58956800 & 4.48633100 & -1.81908900 \\
\hline $\mathrm{H}$ & -1.74270200 & 5.31852200 & -1.66286000 \\
\hline $\mathrm{H}$ & -3.24154300 & 4.44904100 & 0.10612900 \\
\hline $\mathrm{H}$ & -2.42726000 & 2.73717900 & 1.68956900 \\
\hline $\mathrm{H}$ & 3.42010300 & -1.78865600 & 1.73427500 \\
\hline $\mathrm{H}$ & 3.04976200 & -3.26263100 & 0.82689300 \\
\hline $\mathrm{H}$ & 4.64136300 & -2.49695600 & 0.65521200 \\
\hline $\mathrm{H}$ & 4.31009600 & -2.22048200 & -1.93658100 \\
\hline $\mathrm{H}$ & 2.89322600 & -3.23577500 & -1.61878300 \\
\hline $\mathrm{H}$ & 2.70191400 & -1.71947300 & -2.50664600 \\
\hline
\end{tabular}




$\begin{array}{lrrr}\mathrm{H} & 4.98865800 & -0.14029400 & 1.20168900 \\ \mathrm{H} & 5.61097800 & -0.32141300 & -0.45247900 \\ \mathrm{H} & 5.13385500 & 1.27409900 & 0.15096500 \\ \mathrm{H} & 3.46182900 & 1.76661800 & -1.56069300 \\ \mathrm{H} & 4.00712700 & 0.32534600 & -2.43610500 \\ \mathrm{H} & 2.28138200 & 0.54711200 & -2.07306700\end{array}$

